University of Nebraska - Lincoln

DigitalCommons@University of Nebraska - Lincoln

2011

\title{
Theory of the propagation dynamics of spiral edges of diffusion flames in von Kármán swirling flows
}

Javier Urzay

University of California San Diego, jurzay@ucsd.edu

Vedha Nayagam

NASA Glenn Research Center, vedha.nayagam@grc.nasa.gov

Forman A. Williams

University of California San Diego, faw@ucsd.edu

Follow this and additional works at: https://digitalcommons.unl.edu/nasapub

Part of the Physical Sciences and Mathematics Commons

Urzay, Javier; Nayagam, Vedha; and Williams, Forman A., "Theory of the propagation dynamics of spiral edges of diffusion flames in von Kármán swirling flows" (2011). NASA Publications. 94.

https://digitalcommons.unl.edu/nasapub/94

This Article is brought to you for free and open access by the National Aeronautics and Space Administration at DigitalCommons@University of Nebraska - Lincoln. It has been accepted for inclusion in NASA Publications by an authorized administrator of DigitalCommons@University of Nebraska - Lincoln. 


\title{
Theory of the propagation dynamics of spiral edges of diffusion flames in von Kármán swirling flows
}

\author{
Javier Urzay $^{\mathrm{a}, *}$, Vedha Nayagam ${ }^{\mathrm{b}}$, Forman A. Williams ${ }^{\mathrm{a}}$ \\ a Department of Mechanical and Aerospace Engineering, University of California San Diego, La Jolla, CA 92093-0411, USA \\ ${ }^{\mathrm{b}}$ National Center for Space Exploration Research, NASA Glenn Research Center, Cleveland, OH 44135, USA
}

\section{A R T I C L E I N F O}

\section{Article history:}

Received 22 April 2010

Received in revised form 27 June 2010

Accepted 30 August 2010

Available online 13 October 2010

\section{Keywords:}

Edge flames

Triple flames

Non-premixed combustion

Spiral waves

Flame extinction

Swirling flows

\begin{abstract}
A B S T R A C T
This analysis addresses the propagation of spiral edge flames found in von Kármán swirling flows induced in rotating porous-disk burners. In this configuration, a porous disk is spun at a constant angular velocity in an otherwise quiescent oxidizing atmosphere. Gaseous methane is injected through the disk pores and burns in a flat diffusion flame adjacent to the disk. Among other flame patterns experimentally found, a stable, rotating spiral flame is observed for sufficiently large rotation velocities and small fuel flow rates as a result of partial extinction of the underlying diffusion flame. The tip of the spiral can undergo a steady rotation for sufficiently large rotational velocities or small fuel flow rates, whereas a meandering tip in an epicycloidal trajectory is observed for smaller rotational velocities and larger fuel flow rates. A formulation of this problem is presented in the equidiffusional and thermodiffusive limits within the framework of one-step chemistry with large activation energies. Edge-flame propagation regimes are obtained by scaling analyses of the conservation equations and exemplified by numerical simulations of straight two-dimensional edge flames near a cold porous wall, for which lateral heat losses to the disk and large strains induce extinction of the trailing diffusion flame but are relatively unimportant in the front region, consistent with the existence of the cooling tail found in the experiments. The propagation dynamics of a steadily rotating spiral edge is studied in the large-core limit, for which the characteristic Markstein length is much smaller than the distance from the center at which the spiral tip is anchored. An asymptotic description of the edge tangential structure is obtained, spiral edge shapes are calculated, and an expression is found that relates the spiral rotational velocity to the rest of the parameters. A quasiestatic stability analysis of the edge shows that the edge curvature at extinction in the tip region is responsible for the stable tip anchoring at the core radius. Finally, experimental results are analyzed, and theoretical predictions are tested.
\end{abstract}

(c) 2010 The Combustion Institute. Published by Elsevier Inc. All rights reserved.

\section{Introduction}

Swirling flows enhance fuel and oxidizer mixing at the molecular level, promote combustion and flame propagation $[1,2]$ and reduce emissions [3]. Examples of combustion enhancement by increased mixing occur naturally in fire whirls, or in specific engineering designs such as swirl combustors in gas turbines, swirl-generating inlet ports and swirl piston bowls in internal combustion engines. The interaction of flames with swirling air flows in combustion chambers can enhance local extinction for sufficiently large strain rates or heat losses. The boundaries produced by these local extinction phenomena are edge flames, which propagate through the mixture with characteristics similar to those of deflagrations [4]. In swirling boundary layers, local

\footnotetext{
* Corresponding author. Tel.: +1 8585345492.

E-mail addresses: jurzay@ucsd.edu (J. Urzay), vedha.nayagam@grc.nasa.gov (V. Nayagam), faw@ucsd.edu (F.A. Williams).
}

quenching can produce a number of flame patterns and flame fronts, such as straight edge flames, single spiral edge flames, multiple spiral edge flames, flame rings and flame holes, that propagate in the mixture in a nontrivial manner [5-8]. This paper addresses the dynamics and structure of the spiral edge flames found in earlier experiments [5-7]. A snapshot of this type of flame pattern is shown in Fig. 1.

From a broader physical standpoint, spiral patterns are ubiquitous in nature. The Belousov-Zabothinsky reaction [10] and the catalytic surface oxidation of $\mathrm{CO}$ [11] are examples of spiral pattern formation found in physical chemistry. Cell aggregation [12] and calcium waves [13] are examples of spiral patterning in cell signaling, and cardiac fibrillation waves take the form of meandering spirals. However similar at first sight, pattern formation in diffusion flames is a qualitatively and quantitatively different problem than those cited above, which mainly correspond to reactive-diffusive systems [10-13]. Spiral diffusion flames display three-dimensional diffusion and advection effects that make their analytical and 


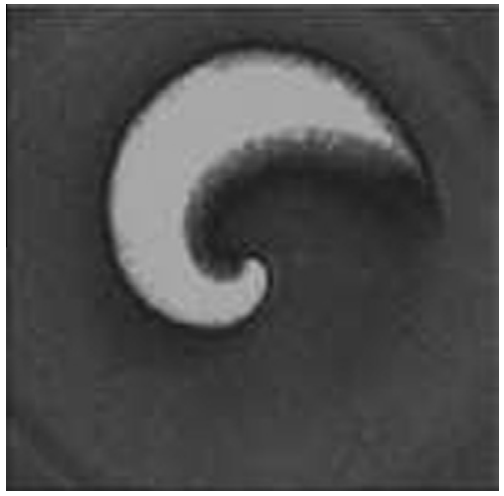

Fig. 1. Spiral edge flame (adapted from [9]).

numerical tractability quite more challenging. Additionally, the strong non-linearities associated with the reaction term in flames are typically exponential in the temperature as in the ZeldovichFrank-Kamenetskii theory [14], which produces sharp interfaces and singular behaviors, whereas classical developments in the reactive-diffusive systems of [10-13] have typically used smoother polynomial non-linearities to account for the propagator source as in the Kolmogorov-Petrovskii-Piskunov theory [15]. Finally, unlike earlier analyses performed on the modelling the physicochemical processes [10-13], the conservation equations and chemical reaction rates used in combustion systems stem from fundamental principles of the kinetic theory of gases, which confers more rigor on the present investigation. Despite all these differences, excitability is a common characteristic shared by all these systems. The concept of excitability of a system regards its ability to trigger abrupt and substantial responses -by means of the autocatalytic production of a propagator or trigger variableto disturbances from a rest state that cross certain characteristic thresholds [16]. After such a response the system momentarily shows a refractory behavior moderated by a controller or refractory variable, in that the system is immune to further stimulation and eventually recovers full excitability.

In the context of flame propagation in stratified mixtures, excitability is related to the reactivity of the mixture and the high sensitivity to temperature because of the effectively high overall activation energy involved in typical combustion chemical reactions. Although the trajectory of a fluid particle occurs generally in a multidimensional space, its initial and final states can be placed on a S-curve response of a diffusion flame [19] as shown in Fig. 2a, which represents the maximum temperature as a function of the reduced Damköhler number $\Delta$, which is defined as

$\Delta=\frac{t_{d}}{t_{c}}$

where $t_{d}$ is the diffusion time through the flame or flame-transit time, and $t_{c}$ is the local chemical time in the flame.

Spatial regions of sufficiently low temperature, in which the mixture remains chemically frozen, may be thought of as a rest state, from which an appreciable excursion may occur when $\Delta$ is sufficiently large to trigger thermal runaway and ignite the mixture. Flame propagation and diffusion of heat into neighboring regions occur once the mixture has been ignited, causing the excitation process to spread spatially into zones initially frozen. For large activation energies and adiabatic systems, the region downstream from the front is close to a Burke-Schumann or equilibrium rest state, involving a reaction zone into which each reactant diffuses and reacts producing a diffusion flame, as in Fig. $2 \mathrm{~b}$. This trailing flame extends infinitely far downstream from the front as in an adiabatic, equidiffusive edge flame or triple flame [4,20-24].

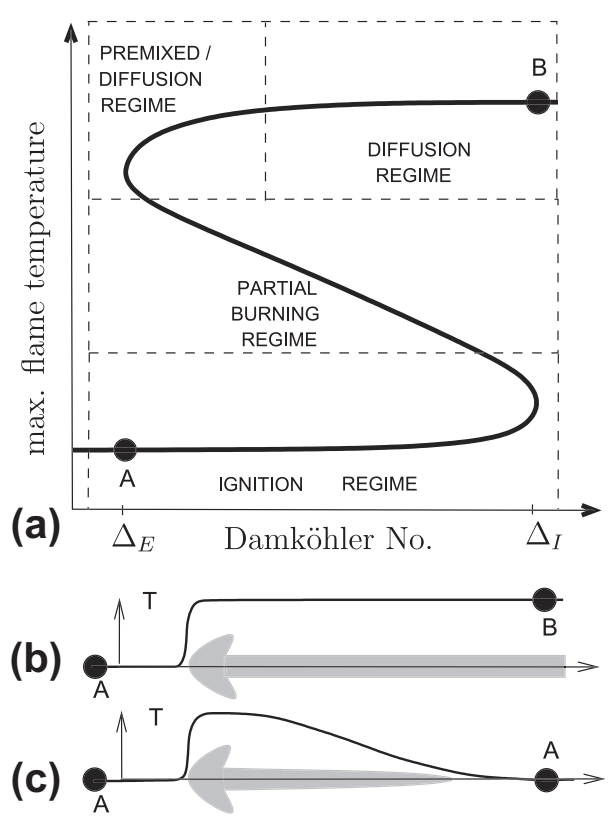

Fig. 2. (a) Schematic representation of the S-curve response of a diffusion flame. The frozen and Burke-Schumann rest states are denoted by A and B, respectively. (b) Temperature profile along the stoichiometric line of a vigorously burning edge flame. (c) Temperature profile along the stoichiometric line of an edge whose diffusion flame has undergone extinction.

For a spiral edge flame, the system achieves the rest state again by means of an extinction tail, as in Fig. 2c. As shown further below, lateral heat losses to the burner surface extinguish the flame when the flame temperature is sufficiently small for the local $\Delta$ to be small, such that the local chemical time becomes large and of the same order as the diffusion time, which produces leakage of unburnt reactants, an associated flame-temperature drop and flame extinction. After extinction, the gaseous mixture is at first refractory to another disturbance, but it finally recovers full ignitability when the reactants are replenished by advection, reproducing the same edge-flame pulse after one revolution provided that no mixing hysteresis has taken place. While this discussion describes how spiral-flame phenomena may be related in general to spiral waves in excitable media, it will be seen later that Fig. 2a does not precisely describe the specific experiments of Fig. 1, in that the indicated ignition event, in fact, is not involved in the physics of the actual process.

Spiral-like flame patterns of different nature than the ones treated in this study have been previously reported for fully premixed combustion systems. Examples of these phenomena are the pelton-like flames found in the combustion of methane and air in radial microchannels [25], the spiraling instabilities observed on the surface of expanding spherical premixed flames in hydrogen-air mixtures [26], and the flames found in the combustion of lean mixtures of butane and oxygen in a pipe [27]. These patterns occur in premixed systems, and their dynamics can be described by twodimensional reaction-diffusion conservation equations.

The paper is organized into five additional sections. Section 2 is dedicated to a general formulation of the problem, in both the laboratory and moving reference frames, and within the framework of a thermodiffusive, equidiffusional model using a single-step chemical reaction of large activation energy. The effects of lateral heat losses to the burner wall on a nearly straight edge flame are addressed in Section 3 by performing scaling analyses of the conservation equations and integrating numerically a two-dimensional model problem. The results in this section do not address the influences of curvature but instead identify limits on the magnitudes of the curvature. Section 4 is dedicated to the study of the tangential 


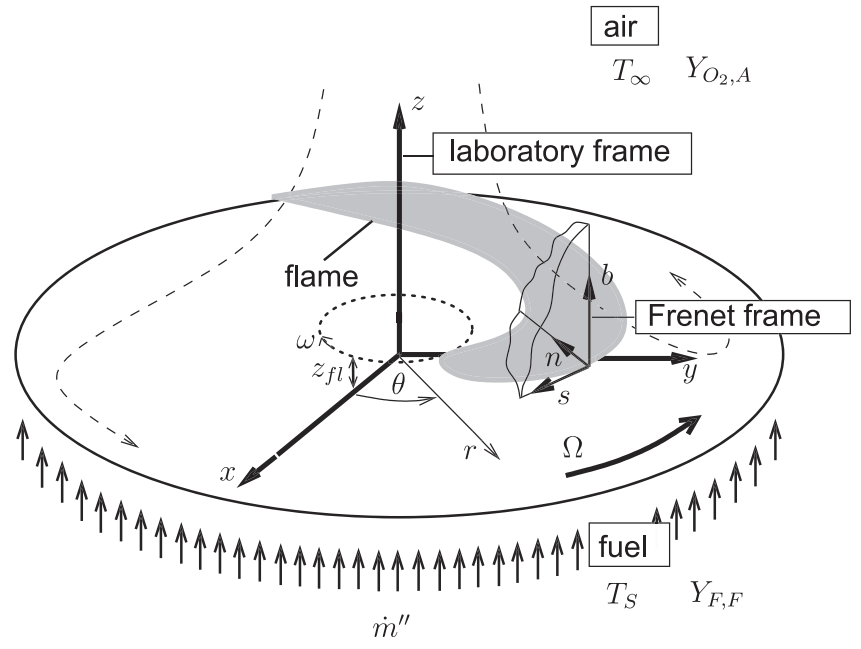

Fig. 3. A schematic diagram of the model problem.

structure of the edge and its propagation in the flow field, here identifying influences of the edge curvature. Finally, a comparison with experimental data is performed in Section 5 and conclusions are drawn in Section 6.

\section{Formulation}

\subsection{Hydrodynamic and mixing fields}

A set of controlled experiments performed with the porous-disk burner demonstrated the existence of a map of flame patterns [5]. The experiments used a sintered bronze, water-cooled porous disk of radius $a=10 \mathrm{~cm}$. Gaseous methane was fed to the disk with uniform and constant fuel flow rate $\dot{m}^{\prime \prime}=0.6-2.0 \mathrm{~g} / \mathrm{sm}^{2}$ from a compressed-gas bottle of fuel mass fraction $Y_{F, F} \approx 1.0$, and injection temperature $T_{S} \approx 300 \mathrm{~K}$ equal to the disk cooling temperature. The exposed disk porous surface was oriented facing downwards to avoid buoyant instabilities, in an otherwise quiescent oxidizing atmosphere of oxidizer mass fraction $Y_{\mathrm{O}_{2}, A} \approx 0.24$, and temperature $T_{\infty} \approx 300 \mathrm{~K}$, and it was spun at a constant angular velocity $\Omega=6$ $50 \mathrm{rad} / \mathrm{s}$. A schematic diagram of the problem is shown in Fig. 3. Further details of the experimental set-up are given in Section 5.

Earlier work [7] proposed a conserved-scalar formulation for this problem that will be followed partially here and that is based on a thermodiffusive and equidiffusional model of the underlying onedimensional diffusion flame. That is, gas densities are assumed constant (a better approximation for flames near walls than for most combustion problems), and species diffusion coefficients are assumed equal to the thermal diffusivity (a better approximation for methane in air than for most fuel-oxidizer systems). The density $\rho$, viscosity $v$, specific heat $c_{p}$, thermal diffusivity $D_{T}$ and Prandtl number $\operatorname{Pr}=v / D_{T}$ are taken to be those of the air side at normal conditions. In the conditions relevant to these experiments, that is at moderately high bulk Reynolds numbers $\operatorname{Re}_{a}=\Omega a^{2} / v \sim 10^{4}$, as the burner rotates the viscous effects are confined to a thin boundary layer of approximate thickness $\delta_{M}=\sqrt{v / \Omega}$ [28], with $\delta_{M} / a \ll 1$. The rotation of the disk induces an entrainment of the ambient fluid with a characteristic velocity $\Omega \delta_{M}$. This fluid is carried by the disk surface through friction and is ejected centrifugally with a characteristic velocity $\Omega \delta_{M} r$, where $r$ is the ratio of the radial coordinate to $\delta_{M}$. This velocity is much larger than the entrainment velocity at sufficiently large radial distances from the center, $r \gg 1$.

In this flow, the resulting dimensionless hydrodynamic velocity field, nondimensionalized with $\Omega \delta_{M}$ in an axial variable $z$ nondimensionalized with $\delta_{M}$, is given by $\mathbf{v}=-\frac{\phi^{\prime}(z) r}{2} \mathbf{e}_{\mathbf{r}}+V(z) r \mathbf{e}_{\theta}+\phi(z) \mathbf{e}_{\mathbf{z}}$

where bold-face characters are vectors in the present notation, $\mathbf{e}_{i}$ representing unit vectors in the subscript direction, and $\phi(z)$ and $V(z)$ are the self-similar nondimensional stream function and azimuth velocity, respectively. These two fundamental functions are shown in Fig. 4 and are obtained by solving numerically the $r$ and $\theta$ components of the momentum conservation equation, $\phi^{\prime \prime \prime}=\phi^{\prime \prime} \phi-\phi^{\prime 2} / 2+2 V^{2}$ and $V^{\prime \prime}=\phi V^{\prime}-\phi^{\prime} V$, subject to non-slip and injection conditions on the disk surface, $\phi^{\prime}=0, V=1$ and $\phi$ $=\mathrm{Re}_{j}$, and similar conditions far from the disk, $\phi^{\prime}=V=0$ and $\phi$ $=\phi_{\infty}$. Fig. 4 shows the velocity profiles obtained from the numerical integration of the momentum conservation equations, along with their asymptotic approximations developed by solving the first two orders of a $\operatorname{Re}_{j} \ll 1$ expansion of the same equations for $z \gg 1$ and $z \ll 1$ [29]. In this formulation, $\phi_{\infty}=O(1)$ is a dimensionless measure of the air entrainment far from the disk and is calculated as part of the hydrodynamic solution, and

$\operatorname{Re}_{j}=\frac{\dot{m}^{\prime \prime}}{\rho \sqrt{v \Omega}}$

is the injection Reynolds number or dimensionless fuel injection rate, defined as the ratio of the injection velocity $\dot{m}^{\prime \prime} / \rho$ to the characteristic entrainment velocity normal to the disk $\delta_{M} \Omega$. The typical values of $\mathrm{Re}_{j}$ encountered in the experiments are found to be much smaller than unity for most of the data range [7], which involves small injection velocities. The strain rate, vorticity and static pressure depend solely on $z$ as a consequence of the radial uniformity of the entrainment. The static pressure is an irrelevant variable that can be eliminated in this thermodiffusive framework. These considerations are not accurate near the disk edge, where

(a)
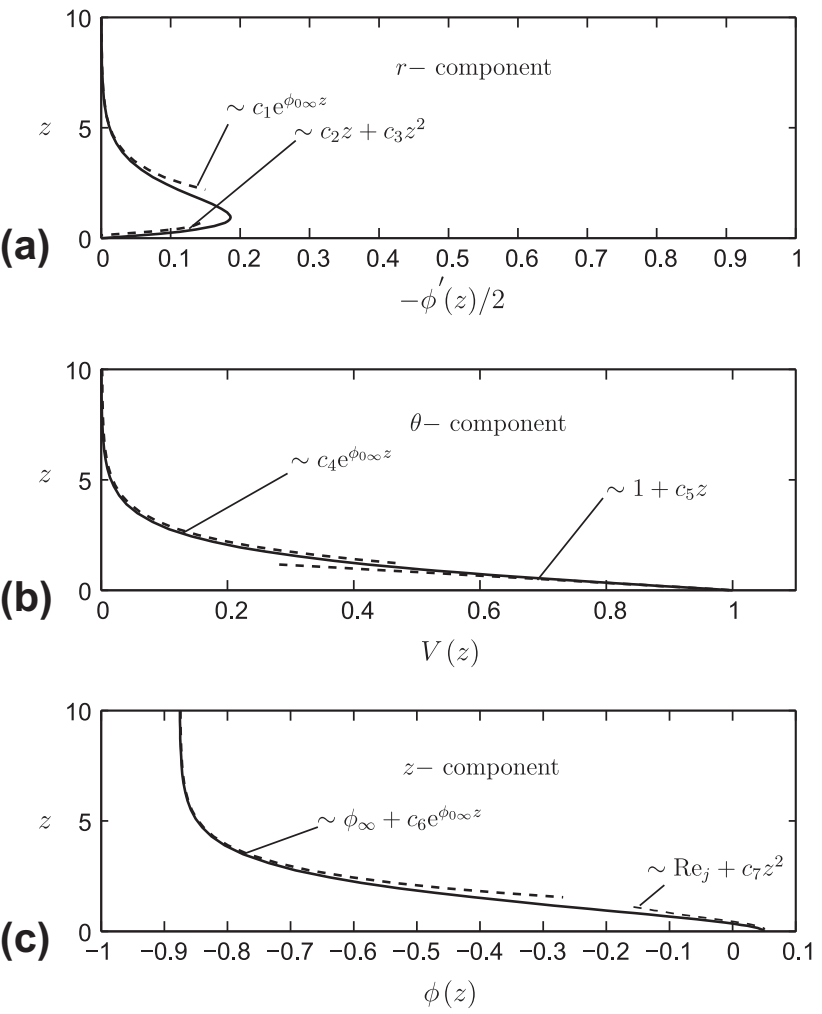

Fig. 4. Numerical (solid line) and asymptotic (dashed line) profiles for the (a) radial velocity, (b) azimuth component, and (c) vertical component of the flow velocity, for $\mathrm{Re}_{j}=0.05$. In this figure, $c_{1}=0.924+2.620 \mathrm{Re}_{j}, \quad c_{2}=0.510+0.046 \mathrm{Re}_{j}$, $c_{3}=-0.500+0.510 \operatorname{Re}_{j}, \quad c_{4}=1.202+2.425 \operatorname{Re}_{j}, \quad c_{5}=-0.616+0.412 \operatorname{Re}_{j}$, $c_{6}=2.091+5.926 \mathrm{Re}_{j}, c_{7}=\operatorname{Re}_{j}, c_{8}=-c_{2}$ and $\phi_{\infty}=\phi_{0 \infty}+0.202 \mathrm{Re}_{j}=-0.884+0.202 \mathrm{Re}_{j}$ are numerically calculated constants. 
the self-similarity imposed by (2) is lost and the velocity normal to the disk and the normal strain rate do not depend solely on the normal coordinate $z$, so that the present calculation implicitly assumes a flow over an infinite disk with uniform air entrainment.

The chemical reaction is considered to be a single-step, secondorder irreversible reaction between methane and the oxygen of the air, $\mathrm{CH}_{4}+2 \mathrm{O}_{2} \rightarrow \mathrm{CO}_{2}+2 \mathrm{H}_{2} \mathrm{O}$, with a dimensionless global rate of reaction

$\hat{\omega}=\operatorname{Da} \widehat{Y}_{F} \widehat{Y}_{\mathrm{O}_{2}} \mathrm{e}^{-T_{a} / T}$.

In this formulation, $\widehat{Y}_{F}$ and $\widehat{Y}_{\mathrm{O}_{2}}$ are the fuel and oxidizer mass fractions normalized with their stoichiometric values $Y_{F, s t}=\alpha Y_{F, F}$ $(1+\alpha)$ and $Y_{\mathrm{O}_{2}, s t}=Y_{\mathrm{O}_{2}, A} /(1+\alpha)$, where $\alpha=Y_{\mathrm{O}_{2}, A} / s Y_{F, F}$ is the mass of the fuel feed stream that is needed to mix with a unit mass of air to generate a stochiometric mixture, and $s$ is the mass of oxidizer burnt per unit mass of fuel consumed in stoichiometric proportions. For methane-air systems and undiluted fuel streams, typical values of $\alpha$ and $s$ are 0.06 and 4 respectively. Similarly, $T$ denotes the temperature nondimensionalized with the adiabatic temperature increment $Q Y_{F, s t} / c_{p}$, where $Q$ is the heat release per unit mass of fuel, and $T_{a}$ represents the corresponding dimensionless activation temperature of the chemical reaction. The parameter Da is a Damköhler number given by

$\mathrm{Da}=\frac{Y_{\mathrm{O}_{2}, \mathrm{t}} \mathrm{A}}{\Omega}$,

which represents the ratio of a pseudo-collision time $\left(Y_{\mathrm{O}_{2}, \mathrm{st}} A\right)^{-1}$ to the flow time $\Omega^{-1}$, with $A$ the frequency factor of the global step.

The mixture fraction $\widetilde{Z}$ is normalized with that of the disk surface $Z_{S}$ and is defined as

$\widetilde{Z}=\widetilde{Z}_{f l}\left(\frac{\widehat{Y}_{F}-\widehat{Y}_{\mathrm{O}_{2}}}{1+\alpha}+1\right), \quad 0 \leqslant \widetilde{Z} \leqslant 1$,

with $\widetilde{Z}=0$ far from the disk and $\widetilde{Z}=1$ on the disk surface. Here $\widetilde{Z}_{f l}=Z_{s t} / Z_{S}$ represents the normalized stochiometric coordinate location with respect to $Z_{S}$, with $Z_{s t}=\alpha /(1+\alpha) \sim 0.056$ the stoichiometric mixture fraction, which determines the diffusion-flame location. The mixing and hydrodynamic fields can be easily related in the present thermodiffusive approximation by making use of (6) in the species conservation equations to integrate a second-order homogeneous differential equation for $\widetilde{Z}(z)$ with appropriate porous-disk boundary conditions, which gives [7]

$\widetilde{Z}(z)=\frac{\operatorname{Re}_{j} \operatorname{Pr}}{\mathcal{B}} \int_{z}^{\infty}\left\{\exp \left[\int_{0}^{\xi} \operatorname{Pr} \phi(z) d z\right]\right\} d \xi$

where $\mathcal{B}$ is an effective mass-transfer number defined as

$\mathcal{B}=\frac{Z_{s t}}{\widetilde{Z}_{f l}-Z_{s t}}=\frac{\alpha\left(1+\alpha+\widehat{Y}_{F, S}-\widehat{Y}_{\mathrm{O}_{2}, S}\right)}{1+\alpha+\alpha\left(\widehat{Y}_{\mathrm{O}_{2}, S}-\widehat{Y}_{F, S}\right)}$,

and the subindex $S$ refers to the fuel and oxidizer mass fractions on the disk surface.

The effective mass-transfer number increases monotonically with $\mathrm{Re}_{j}$. If there is no fuel injected, $\operatorname{Re}_{j}=0, \widetilde{Z}_{f l} \rightarrow \infty, Z_{S}=0$, $Y_{F, S}=0, Y_{\mathrm{O}_{2}, S}=Y_{\mathrm{O}_{2}, A}$ and $\mathcal{B}=0$. Similarly, for large injection rates, $\operatorname{Re}_{j} \rightarrow \infty, \widetilde{Z}_{f l} \rightarrow Z_{s t}, Z_{S} \rightarrow 1, Y_{F, S} \rightarrow Y_{F, F}, Y_{\mathrm{O}_{2}, S} \rightarrow 0$ and $\mathcal{B} \rightarrow \infty$. From (6)-(8), an equivalent definition

$\mathcal{B}=\operatorname{Re}_{j} \operatorname{Pr} \int_{0}^{\infty}\left\{\exp \left[\int_{0}^{\xi} \operatorname{Pr} \phi(z) d z\right]\right\} d \xi$

is found. The result (9) can be simplified for small values of $\operatorname{Re}_{j}$ by expanding the self-similar stream function and azimuth velocity in powers of $\mathrm{Re}_{j}$ and numerically solving each order of the $r$ and $\theta$ momentum equations [29]. This procedure gives the expansion $\mathcal{B} \sim \mathcal{B}_{0} \operatorname{Re}_{j}+\mathcal{B}_{1} \operatorname{Re}_{j}^{2}$, with $\mathcal{B}_{0}=2.180$ and $\mathcal{B}_{1}=2.349$. These results for $\widetilde{Z}$ and $\mathcal{B}$ are plotted in Fig. 5a and $b$.
The squared gradient of the mixture fraction multiplied by the thermal diffusivity is commonly referred to as the scalar dissipation rate $\chi$ in turbulent combustion [2], and it represents the inverse of a characteristic diffusion time. When the diffusion time is evaluated in the burning diffusion flame, $\widetilde{Z}=\widetilde{Z}_{f l}$, a characteristic flame-transit time or reactant diffusion time through the flame $t_{d} \sim 1 / \chi_{f l}$ is obtained, which is usually large compared to the local chemical time $t_{c} \sim\left(A Y_{\mathrm{O}_{2}, s t}\right)^{-1} \exp \left(T_{a} / T\right)$ to ensure complete reactant depletion. Here the same nomenclature is used, and the expression for the nondimensional scalar dissipation rate

$\widetilde{\chi}(z)=\left(\frac{d \widetilde{Z}}{d z}\right)^{2}=\left(\frac{\operatorname{PrRe}_{j}}{\mathcal{B}}\right)^{2} \exp \left[2 \int_{0}^{z} \operatorname{Pr} \phi(\xi) d \xi\right]$

is found, where use has been made of (7). The value of $\tilde{\chi}$ on the disk surface is given by $\widetilde{\chi}_{s}=\left(\operatorname{PrRe}_{j} / \mathcal{B}\right)^{2}$. For small mixture fractions $\widetilde{Z} \rightarrow 0(z \rightarrow \infty)$, the approximation $\phi \sim \phi_{\infty}$ in (7) yields $\widetilde{\chi}(\widetilde{Z}) \sim$ $\left(\phi_{\infty} \operatorname{Pr} \widetilde{Z}\right)^{2}$. Similarly, for $\widetilde{Z} \sim 1(z \rightarrow 0)$, the approximation $\phi \sim \operatorname{Re}_{j}$ gives $\widetilde{\chi}(\widetilde{Z}) \sim \widetilde{\chi}_{S}\left[1-3(\widetilde{Z}-1) \operatorname{Pr} \operatorname{Re}_{j} / \widetilde{\chi}_{s}^{1 / 2}\right]^{2 / 3}$. The distribution of $\widetilde{\chi}$ is shown in Fig. $5 \mathrm{c}$. In this notation, the flame transit time is given by $t_{d} \sim \delta_{M}^{2} /\left(D_{T} Z_{S}^{2} \widetilde{\chi}_{f l}\right)$, which decreases with increasing mass flow rate and angular velocity.

An excess enthalpy $H$, nondimensionalized with the chemical heat release $Q Y_{F, s}$, can be defined as

$H=\frac{\alpha \widehat{Y}_{F}+\widehat{Y}_{\mathrm{O}_{2}}}{1+\alpha}-1+T-T_{\infty}$,

with $H=0$ in the oxidizer feed stream, and $H=H_{S}$ on the disk surface, where in the most general case $H_{S}$ varies with time and position on the disk surface in a manner that is calculated as part of the solution. This excess enthalpy is zero everywhere for an equidiffusive counterflow diffusion flame with equal thermal enthalpies of oxidizer and fuel feed streams [19]. The excess enthalpy in the present analysis is, however, non-zero even in the case of equal feedstream temperatures, $T_{S}=T_{\infty}$, because of heat losses to the disk.
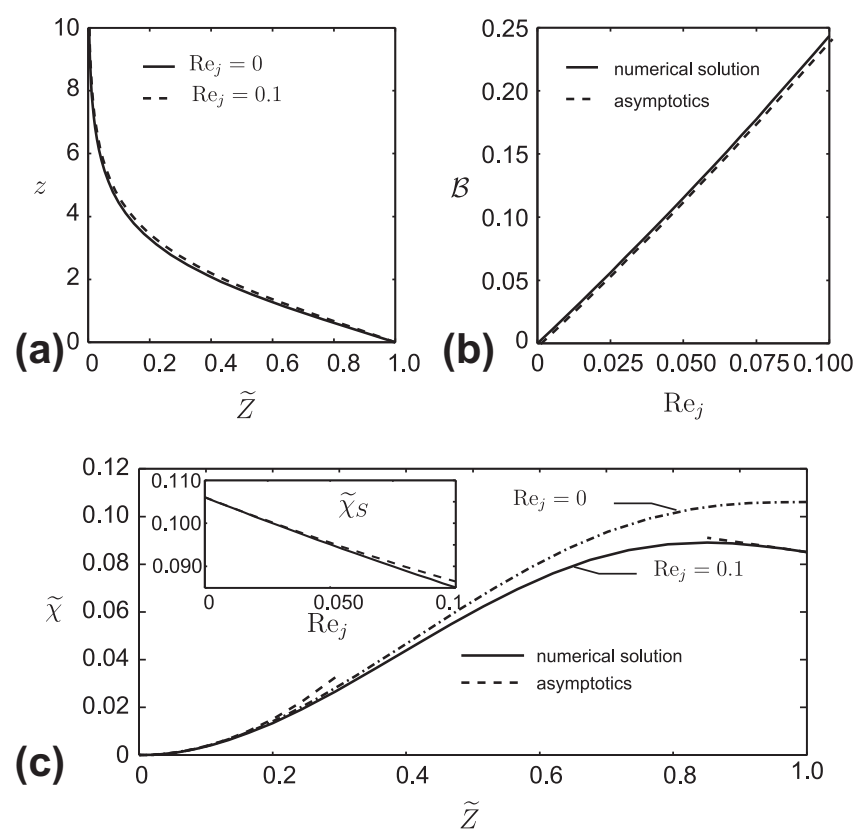

Fig. 5. (a) Mixture fraction distribution (7), (b) mass-transfer number (9), and (c) dimensionless scalar dissipation rate (10). The inset shows the dimensionless scalar dissipation rate on the disk surface. 


\subsection{Laboratory-frame formulation}

In these variables and in the laboratory reference frame, the species and energy conservation equations become

$\frac{\partial H}{\partial t}+\mathbf{v}_{\perp} \cdot \nabla_{\perp} H=\frac{\nabla_{\perp}^{2} H}{\operatorname{Pr}}+\frac{\tilde{\chi}(\widetilde{Z})}{\operatorname{Pr}} \frac{\partial^{2} H}{\partial \widetilde{Z}^{2}}$,

$\frac{\partial T}{\partial t}+\mathbf{v}_{\perp} \cdot \nabla_{\perp} T=\frac{\nabla_{\perp}^{2} T}{\operatorname{Pr}}+\frac{\widetilde{\chi}(\widetilde{Z})}{\operatorname{Pr}} \frac{\partial^{2} T}{\partial \widetilde{Z}^{2}}+\operatorname{Da} \widehat{Y}_{F} \widehat{Y}_{\mathrm{O}_{2}} \mathrm{e}^{-T_{a} / T}$,

$\widehat{Y}_{F}=H+\widetilde{Z} / \widetilde{Z}_{f l}+T_{\infty}-T$,

$\widehat{Y}_{\mathrm{O}_{2}}=1+\alpha\left(1-\widetilde{Z} / \widetilde{Z}_{f l}\right)+H+T_{\infty}-T$,

subject to

$H+\frac{\beta}{Z_{s t}}+\frac{1}{\mathcal{B}} \frac{\partial H}{\partial \widetilde{Z}}=0, \quad T=T_{S}$,

at $\widetilde{Z}=1$

$H \rightarrow 0, \quad T \rightarrow T_{\infty}$

at $\widetilde{Z}=0$,

$\frac{\partial H}{\partial r} \rightarrow 0, \quad \frac{\partial T}{\partial r} \rightarrow 0$,

for $r \rightarrow \infty$, and

$\left.\frac{\partial H}{\partial \theta}\right|_{\theta=0}=\left.\frac{\partial H}{\partial \theta}\right|_{\theta=2 \pi},\left.\quad \frac{\partial T}{\partial \theta}\right|_{\theta=0}=\left.\frac{\partial T}{\partial \theta}\right|_{\theta=2 \pi}$,

for $0 \leqslant r<\infty$ and $0 \leqslant \widetilde{Z} \leqslant 1$. In this formulation, the time coordinate $t$ is nondimensionalized with the flow time $\Omega^{-1}$, and $\nabla_{\perp}$ and $\mathbf{v}_{\perp}$ are the two-dimensional gradient operator and two-dimensional hydrodynamic velocity field in $r$ and $\theta$. The boundary condition (16) represents a combination of reactant mass conservation and thermal contact along the disk surface, with

$\beta=Z_{s t}\left\{\left(T_{\infty}-T_{S}\right)-\left.\frac{1}{\mathcal{B}} \frac{\partial T}{\partial \widetilde{Z}}\right|_{\widetilde{Z}=1}\right\}$,

a heat-loss coefficient that accounts for feed-stream enthalpy differences and heat losses to the disk surface, the behavior of which was analyzed in earlier work for a uniform diffusion flame enveloping the entire disk [7]. The coefficient $\beta$ generally decreases with increasing $\mathrm{Re}_{j}$ for subadiabatic disk temperatures [7], with $\beta \rightarrow Z_{s t}\left(T_{\infty}-T_{S}\right)$ for $\operatorname{Re}_{j} \gg 1$.

Eqs. (2), (7), (9), (10) and (12)-(20) give rise to the three-dimensional solution of the temperature and excess-enthalpy distributions in the physical space and in the laboratory reference frame. The numerical integration of an equivalent set of conservation equations in a non-conserved scalar form was performed in earlier work [8] to capture spiral flames with reasonable success given the difficulty of such three-dimensional and intensive calculation, although spiral meandering and solid-rigid rotation around a central hole were not obtained, thus contradicting existing experiments (perhaps due to an inaccurate resolution of the vicinity of the burner axis), and analyses of the front and trailing diffusionflame structures and their propagation dynamics were not performed. Nonetheless, that study is valuable in that it confirms that all the essential physics is contained within the set of equations outlined above.

\subsection{The frozen and Burke-Schumann regimes}

The problem outlined in the preceding section can be solved analytically in the frozen and Burke-Schumann regimes, $\Delta \ll 1$ and $\Delta \gg 1$ respectively, where $\Delta$ is the reduced Damköhler number given by (1).
In the frozen limit, the diffusion time is much smaller than the local chemical time, $\Delta \ll 1$, the reaction term is negligible in the first approximation, and (12) and (13) reduce to $d^{2} H / d \widetilde{Z}^{2}=$ $d^{2} T / d \widetilde{Z}^{2}=0$ subject to (16) and (17), which gives

$T=T_{\infty}-\beta_{f r} \widetilde{Z} / \widetilde{Z}_{f l}$,

$H=-\beta_{f r} \widetilde{Z} / \widetilde{Z}_{f l}$,

$\widehat{Y}_{F}=\widetilde{Z} / \widetilde{Z}_{f l}$,

$\widehat{Y}_{\mathrm{O}_{2}}=1+\alpha\left(1-\widetilde{Z} / \widetilde{Z}_{f l}\right)$,

where $\beta_{f r}$ denotes the heat loss coefficient (20) in the frozen regime based on the frozen temperature profile,

$\beta_{f r}=\widetilde{Z}_{f l}\left(T_{\infty}-T_{S}\right)$.

The value $H_{f r}=-\beta_{f r}$ represents the excess enthalpy evaluated at the stoichiometric coordinate. Because of the boundary condition at the disk surface, this excess enthalpy is negative if the plate temperature is less than that of the air and positive if it is greater. Further increase in $\Delta$ to a critical $\Delta_{I}$ by, for instance, decreasing the angular velocity or increasing the disk temperature, gives rise to higher-order corrections to (21)-(24), which are representative of flame autoignition as depicted in Fig. 2a.

In the Burke-Schumann limit, the local chemical time is much shorter than the diffusion time through the flame, $\Delta \gg 1$, so that the flow remains in chemical equilibrium on both sides of a thin reaction sheet of infinitesimal thickness, which is much smaller than the hydrodynamic length scale, and no reactant leakage occurs in the first approximation. Thus, (12) and (13) reduce to $d^{2} H / d \widetilde{Z}^{2}=0$ and $d^{2} T / d \widetilde{Z}^{2}=-\operatorname{Pr} \hat{\omega} / \widetilde{\chi}$, with $\hat{\omega}$ approximated as a Dirac delta function. The solution to these equations is

$T=T_{\infty}+1+\alpha-\left(\alpha+\beta_{f l}\right) \widetilde{Z} / \widetilde{Z}_{f l}$,

$Y_{F}=(1+\alpha)\left(\widetilde{Z} / \widetilde{Z}_{f l}-1\right), \quad Y_{O_{2}}=0$,

in the fuel region $\widetilde{Z}_{f l} \leqslant \widetilde{Z} \leqslant 1$,

$T=T_{\infty}+\left(1-\beta_{f l}\right) \widetilde{Z} / \widetilde{Z}_{f l}$,

$Y_{\mathrm{O}_{2}}=(1+\alpha)\left(1-\widetilde{Z} / \widetilde{Z}_{f l}\right), \quad Y_{F}=0$,

in the oxidizer region $0 \leqslant \widetilde{Z} \leqslant \widetilde{Z}_{f l}$, and

$H=-\beta_{f l} \widetilde{Z} / \widetilde{Z}_{f l}$,

with

$\beta_{f l}=\beta_{f r}+\alpha / \mathcal{B}$,

the heat-loss coefficient (20) based on equilibrium conditions. The value $H_{f l}=-\beta_{f l}$ corresponds to the excess enthalpy in the diffusion flame. The excess enthalpy in the Burke-Schumann limit is always more negative than that in the frozen limit for subadiabatic disk temperatures because of the enhanced heat loss to the porous plate caused by the heat conduction from the hot flame.

Further decrease of $\Delta$ below a critical $\Delta_{E}$ by, for instance, increasing the angular velocity or decreasing the fuel flow rate, gives rise to higher-order corrections to (26)-(30) that are representative of flame extinction, as depicted in Fig. 2a. An expression for the critical Damköhler number of extinction was derived in earlier work [7], which is reproduced here for illustrative purposes and for future use,

$\Delta_{E}=\frac{4 \operatorname{Pr} \widetilde{Z}_{f l}^{2}\left(T_{f l}^{2} / T_{a}\right)^{3}}{\widetilde{\chi}_{f l}(1+\alpha)^{2}} \mathrm{Dae}^{-T_{a} / T_{f l}}$,

where $T_{f l}$ is the non-adiabatic Burke-Schumman temperature

$T_{f l}=T_{\infty}+1-\beta_{f l}$,

of the diffusion flame, which decreases with decreasing Reynolds numbers of injection. Additionally, a solvability condition for the 
non-adiabatic Burke-Schumann solution was obtained [7], in that mass-transfer numbers above a minimum $\mathcal{B}^{\text {min }}=\alpha$ must occur for the Burke-Schumann flame to stand above the disk surface, since smaller values of the mass-transfer number $\mathcal{B}<\mathcal{B}^{\text {min }}$ would not be capable of generating stoichiometric mixtures outside the disk. When use is made of the small- $\mathrm{Re}_{j}$ asymptotic expansion for $\mathcal{B}$, the minimum injection Reynolds number corresponding to $\mathcal{B}^{\min }$ is found to be $\operatorname{Re}_{j}^{\min } \sim 0.027$.

At sufficiently large $\mathrm{Re}_{\mathrm{j}}$, the influences of the disk surface are negligible, and the adiabatic flame temperature

$T_{f l}^{\infty}=1+T_{\infty}-Z_{s t}\left(T_{\infty}-T_{S}\right)$

is recovered in (33). In this limit, the excess-enthalpy distribution is solely produced by feed-stream enthalpy differences,

$H=-Z\left(T_{\infty}-T_{S}\right)$,

which then holds for both frozen and Burke-Schumann regimes.

\subsection{Frenet-frame formulation}

The advancing edge region of the flame is a zone of finite thickness $\delta_{f}$. Nevertheless, an edge location can be defined in a precise manner, for example, as the locus of the inflection points of the temperature profile, or, for large activation energies, as a discontinuity in a much larger scale, when the edge reaction zone is very thin compared with the hydrodynamic length scale of the flow, $\delta_{f} / \delta_{M} \ll 1$. An orthogonal, right-handed Frenet frame $\{n, s, b\}$ is defined attached to the moving curvilinear edge as depicted in Fig. 3. The Frenet trihedron is defined by the unit tangent vector $\mathbf{e}_{\mathbf{s}}$ to the edge resulting from the intersection of the osculating and rectifying planes, the unit normal vector $\mathbf{e}_{\mathbf{n}}$ to the edge resulting from the intersection of the osculating and binormal planes, and the unit binormal vector $\mathbf{e}_{\mathbf{b}}$ to the edge resulting from the intersection of the binormal and rectifying planes. The normal vector $\mathbf{e}_{\mathbf{n}}$ is defined as positive when pointing towards the burnt side of the edge, so that the front curvature $\mathcal{K}_{s}$ is then positive for fronts concave towards the flame sheet to satisfy by construction the first Frenet-Serret formula $d \mathbf{e}_{\mathbf{s}} / d s=\mathcal{K}_{s} \mathbf{e}_{\mathbf{n}}$ [30]. Additionally, the edge is assumed to have zero torsion, so that the third Frenet-Serret formula yields $d \mathbf{e}_{\mathbf{b}} / d s=0$, with $\mathbf{e}_{\mathbf{b}}=\mathbf{e}_{\mathbf{z}}$. Therefore, the equation of the osculating plane of the trailing flame is simply given by $z=z\left(\widetilde{Z}_{f l}\right)$ in (7), with the edge-flame propagating along lines of stoichiometric mixture.

The edge may mathematically be described by introducing the scalar $G(x, y, t)=G_{0}$, with $G<G_{0}$ and $G>G_{0}$ for the burned and unburned states, respectively. If the surface is smooth and continuous, the previously defined unit vector normal to the edge is then given by

$\mathbf{e}_{\mathbf{n}}=-\nabla G /|\nabla G|$.

Every Lagrangian edge element may be considered to move with a velocity $\mathbf{U}_{\mathbf{L F}}$ with respect to the laboratory frame, and since each such element remains on the edge, the relation

$\frac{d G}{d t}=\frac{\partial G}{\partial t}+\mathbf{U}_{\mathbf{L F}} \cdot \nabla G=0$

serves to maintain the value of $G$ equal to $G_{0}$ on the edge at future times. Although the previously defined nondimensionalization for lengths, times and velocities are applied here, the symbols also could be considered to represent dimensional quantities in (37), and different, appropriately revised nondimensionalizations will be introduced later. The relationship (37), which lies at the basis of level-set methods, is to be used here for edge-tracking purposes. Clearly, only the component of $\mathbf{U}_{\mathbf{L F}}$ in the $\mathbf{e}_{\mathbf{n}}$ direction is relevant, whence the tangential component can be chosen arbitrarily, a freedom which has been applied in an earlier study of the time-depen- dent stability of reactive-diffusive spiral waves to derive a convenient formulation [31].

Daou and Liñán [24] have shown that the ratio of a characteristic flame thickness $\delta_{L}^{0}=D_{T} / S_{L, s t}^{0}$ of a laminar planar premixed flame to the radius of curvature of the premixed wings $\epsilon \delta_{M}$, namely

$\Gamma=\frac{\delta_{L}^{0}}{\epsilon \delta_{M}}$

is of paramount importance for characterizing the structure and propagation dynamics of a triple flame. An edge flame represents the limit structure of a very slender triple flame propagating in large mixture-fraction gradients, $\quad \Gamma \rightarrow \infty$. In this formulation, $\mathrm{S}_{L, s t}^{0}=\left(4 \epsilon^{3} A D_{T} Y_{\mathrm{O}_{2}, s t} \mathrm{e}^{-T_{a} / T_{f}}\right)^{1 / 2}$ is the burning rate of a planar laminar premixed flame in stoichiometric proportions when the reaction rate is given by (4), $T_{f}$ is a characteristic nondimensional edge temperature, and $\epsilon$ is the inverse of a Zel'dovich number given by

$\epsilon=T_{f}^{2} / T_{a} \ll 1$,

which is usually a small parameter in combustion applications because of the large overall activation energies involved in typical combustion processes. The propagation parameter $\Gamma$ is related to a reduced Damköhler number $\Delta_{f}$ of the edge based on the temperature of the edge,

$\Delta_{f}=\left(\frac{\delta_{M}}{\delta_{L}^{0}}\right)^{2}=4 \operatorname{Pr} \epsilon^{3} \mathrm{Dae}^{-T_{a} / T_{f}}=\left(\frac{S_{L, s t}^{0}}{\operatorname{Pr} \delta_{M} \Omega}\right)^{2}=\frac{1}{\Gamma^{2} \epsilon^{2}}$,

which represents the ratio of the diffusion time through the mixing layer to the flame transit time over the front thickness. The reduced Damköhler number is also related to the Karlovitz number Ka or dimensionless strain rate, $\Delta_{f}=1 / \mathrm{Ka}$.

For large activation energies, $\epsilon \ll 1$, and low tangential curvatures, the length scale of variations in the normal direction to the front is much smaller than along its tangential direction. Thus, the edge-flame temperature and excess enthalpy have a weak dependence -of $O(\epsilon)$ at most- on the $s$ coordinate in the first approximation, and solutions of (12) and (13) are sought for which contours $n=$ constant are level curves of temperature and excess enthalpy. Additionally, if the edge is not deforming too rapidly, the time derivative in the Frenet frame can be neglected and translation-invariant solutions can be obtained; this occurs for a slowly meandering and slowly varying edge flame in which the time scale of variation in the moving frame is much larger that $1 / \Omega$. When the radius of curvature of the premixed wings $\epsilon \delta_{M}$ is used as the unit length of the normal coordinate $n$, the planar premixed flame velocity $S_{L, s t}^{0}$ is used as the unit velocity scale, and the mixture fraction coordinate is stretched about the diffusion-flame height as $\widetilde{Z}^{\star}=\left(\widetilde{Z}-\widetilde{Z}_{f l}\right) / \epsilon$, Eqs. (12)(17) become

$$
\begin{aligned}
{\left[\mathrm{U}_{\mathrm{n}}+\Gamma \epsilon \mathcal{K}_{s}+\mathrm{v}_{\mathrm{n}, \mathrm{rel}}\left(\widetilde{Z}^{\star}\right)\right] \frac{\partial H}{\partial n}=} & \Gamma \frac{\partial^{2} H}{\partial n^{2}}+\Gamma \widetilde{\chi}\left(\widetilde{Z}^{\star}\right) \frac{\partial^{2} H}{\partial \widetilde{Z}^{\star}} \\
{\left[\mathrm{U}_{\mathrm{n}}+\Gamma \epsilon \mathcal{K}_{s}+\mathrm{v}_{\mathrm{n}, \mathrm{rel}}\left(\widetilde{Z}^{\star}\right)\right] \frac{\partial T}{\partial n}=} & \Gamma \frac{\partial^{2} T}{\partial n^{2}}+\Gamma \widetilde{\chi}\left(\widetilde{Z}^{\star}\right) \\
& \times \frac{\partial^{2} T}{\partial \widetilde{Z}^{\star}},+\frac{\widehat{Y}_{F} \widehat{Y}_{\mathrm{O}_{2}}}{4 \Gamma \epsilon^{3}} \\
& \times \exp \left[-\frac{1}{\epsilon} \frac{\left(T_{f}-T\right)}{T / T_{f}}\right]
\end{aligned}
$$

and

$\widehat{Y}_{F}=H+\epsilon \widetilde{Z}^{\star} / \widetilde{Z}_{f l}+1+T_{\infty}-T$,

$\widehat{Y}_{\mathrm{O}_{2}}=1-\alpha \epsilon \widetilde{Z}^{\star} / \widetilde{Z}_{f l}+H+T_{\infty}-T$, 
subject to

$\epsilon\left(H+T_{\infty}-T_{S}\right)+\frac{1}{\mathcal{B}}\left(\frac{\partial H}{\partial \widetilde{Z}^{\star}}-\frac{\partial T}{\partial \widetilde{Z} \star}\right)=0, \quad T=T_{S}$,

at $\widetilde{Z}^{\star}=\left(1-\widetilde{Z}_{f l}\right) / \epsilon$, and

$H \rightarrow 0, \quad T \rightarrow T_{\infty}$

at $\widetilde{Z}^{\star}=-\widetilde{Z}_{f l} / \epsilon$. In this formulation,

$\mathrm{U}_{\mathrm{n}}=\left(\left.\mathbf{v}_{\perp}\right|_{\tilde{Z}=\tilde{Z}_{f l}}-\mathbf{U}_{\mathbf{L F}}\right) \cdot \mathbf{e}_{\mathbf{n}}$

is the front propagation velocity or burning-rate eigenvalue (in general dependent on the tangential curvature of the edge), and

$\mathcal{K}_{s}=-\nabla \cdot \mathrm{e}_{\mathrm{n}}=\frac{\nabla^{2} G+\mathbf{e}_{\mathbf{n}} \cdot \nabla\left(\mathbf{e}_{\mathbf{n}} \cdot \nabla G\right)}{|\nabla G|}$

is the edge tangential curvature measured in the hydrodynamic scale $1 / \delta_{M}$. Eqs. (41)-(46) also assume small tangential curvatures, $\mathcal{K}_{s} \ll \mathrm{U}_{\mathrm{n}}^{0} / \Gamma \epsilon$, such that the tangential variations of the temperature and excess enthalpy are $\leqslant O(\epsilon)$; for $\mathcal{K}_{s} \gg \mathrm{U}_{\mathrm{n}}^{0} / \Gamma \epsilon$ the tangential heat losses become important. Eqs. (41) and (42) also contain the quantity $\mathbf{v}_{\mathrm{n}, \text { rel }}=\left(\mathbf{v}_{\perp}-\left.\mathbf{v}_{\perp}\right|_{\widetilde{Z}=\widetilde{Z}_{f}}\right) \cdot \mathbf{e}_{\mathbf{n}}$, which represents the relative flow advection with respect to the edge.

Upstream from the edge, $n \rightarrow-\infty$, the temperature and excessenthalpy profiles correspond to the frozen profiles, (21) and (22). Downstream from the edge, $n \rightarrow+\infty$, the temperature and excess-enthalpy profiles can correspond either to the equilibrium profiles, (26), (28) and (30), or to the frozen profiles, (21) and (22), depending on the value of the mass-transfer number $\mathcal{B}$ as detailed further below.

\section{Influences of heat losses to the burner surface on the propagation of edge flames}

Order-of-magnitude analyses can be performed in Eqs. (41)(46) as follows. The propagation velocity $U_{n}^{0}$, the value of $U_{n}$ for zero tangential curvature $\left(\mathcal{K}_{s}=0\right)$, nondimensionalized with $S_{L . s t}^{0}$, is of $O(1)$ as observed from a convective-diffusive balance in the preheat region, which has a thickness ${ }^{1}$ of $O(\Gamma \epsilon)$. In this region, in (41) and (42) both streamwise convection and diffusion terms are of $O(1 / \Gamma)$, whereas the transverse diffusion and reaction terms are of $O\left(\Gamma \epsilon^{2}\right)$ and $O\left(\mathrm{e}^{-1 / \epsilon}\right)$, respectively, the former following from $\tilde{\chi}$ and changes in $\widetilde{Z}$ being of $O(1)$ and the latter from $T_{f}-T$ being of $O(1)$. The nondimensional reaction-layer thickness $\delta_{f}$ in the edge region, where variations in temperature and composition are of $O(\epsilon)$, is obtained by a balance between the streamwise diffusion and reaction terms in (41) and (42), $\delta_{f} \sim \Gamma \epsilon^{2}$. Thus, the streamwise diffusion and reaction terms in the edge reaction-layer region are of $O(1 / \Gamma \epsilon)$ in (41) and (42), whereas the streamwise convection transport is of $O(1 / \Gamma)$ and the transversal diffusion term is of $O(\Gamma \epsilon)$, since variations of temperature of $O(\epsilon)$ occur in transversal distances of $O(\epsilon)$, which are of the same order as the radius of curvature $\epsilon$ of the triple-flame wings. The thickness of the trailing diffusion flame $\delta_{f l}$, where variations in temperature and composition are of order $\epsilon \Delta_{f l}^{-1 / 3}$, is obtained by a balance between the transversal diffusion and reaction terms, $\delta_{f l} \sim \epsilon \Delta_{f l}^{-1 / 3}$. Finally, if $\Delta_{f l} \sim \Delta_{f}$, the diffusionflame Damköhler number can be related to the propagation parameter $\Gamma$ by using (40). However, both Damköhler numbers can differ in large amounts, as detailed below. These estimates are used in what follows to obtain the characteristic dimensions of triple flames propagating in strained mixing layers in both adiabatic and non-adiabatic modes.

${ }^{1}$ Characteristic thicknesses in this discussion are normalized with the mixing-layer thickness $\delta_{M}$
Numerical computations were performed to analyze and isolate the effects of the cold porous wall on the propagation of a nearly straight edge flames. For that purpose, Eqs. (41)-(46) were integrated numerically for zero tangential curvature $\left(\mathcal{K}_{s}=0\right)$, and without streamwise hydrodynamic flow effects, $\mathrm{v}_{\mathrm{n}, \mathrm{rel}}=0$ (a better approximation for smaller values of $\Gamma$ ), subject to (45) on the disk surface, to the frozen profiles (21)-(24) far upstream from the edge and to zero excess enthalpy (46) in the oxidizer stream far from the surface. The effect of the von Kármán flow that was retained is its influences on the mixture-fraction field, through the scalar dissipation rate (10), which appears in (41) and (42). The boundary conditions far downstream were calculated from the conservation equations in each iteration. A schematic diagram of the computational model is shown in Fig. 6.

Daou and Liñán [24] performed similar calculations for a straight, two-dimensional steady triple flame in a steady, planar, two-dimensional counterflow mixing layer. That flow configuration is similar to the adiabatic (large- $\mathcal{B}$ ) limit of the present model, differing only in the functional form of the scalar dissipation rate $\tilde{\chi}$, and the similarity of the results helps to validate the numerical code utilized here. In the present work, an adiabatic calculation, similar to the calculation of [24], was made using a Zel'dovich number $1 / \epsilon=8$ and a stoichiometric mixture fraction $Z_{s t}=1 / 2$, with a single-step chemical reaction of pure Arrhenius type and zero feed-stream temperature difference $T_{\infty}=T_{S}$. The numerical calculations for the non-adiabatic case (small- $\mathcal{B}$ limit) used Eqs. (41)-(46) with the von Kármán scalar dissipation rate (10), the same Zel'dovich number as in the adiabatic case, and a $\mathrm{CH}_{4}$-air stoichiometric mixture fraction $Z_{s t}=0.056$, with zero feed-stream temperature difference $T_{\infty}=T_{S}$, and variable activation energy and heat release as in [32] to avoid unrealistic super-adiabatic flame speeds on the fuel side that are artifacts of hydrocarbon single-step reactions with constant activation energy [32].

Both cases were calculated using a centered, second-order finite-differences method with an explicit predictor-corrector time-relaxation numerical scheme, and with 42,000 mesh points for the adiabatic case and 72,000 mesh points for the non-adiabatic case. Numerical convergence was established by obtaining a constant propagation velocity and time-invariant excess enthalpy and temperature distributions with a tolerance of $10^{-8}$. The method for anchoring the flame within the computational domain and for obtaining the burning-rate eigenvalue closely followed earlier work [33]. First, the computation was performed in the laboratory frame with a localized ignition of the mixture far downstream from the anchoring point, and the resulting propagation velocity was observed. Next, the front was fixed in a moving frame by specifying the temperature at an arbitrary anchoring point (which in the calculations was set to $n=0$ and $Z=Z_{\text {st }}$ ), and the previously observed velocity was used as a first approximation in an iterative process. Finally, the convection velocity in the moving frame was varied until convergence of the steady-flow problem was achieved. The results will be described after the propagation regimes are identified.

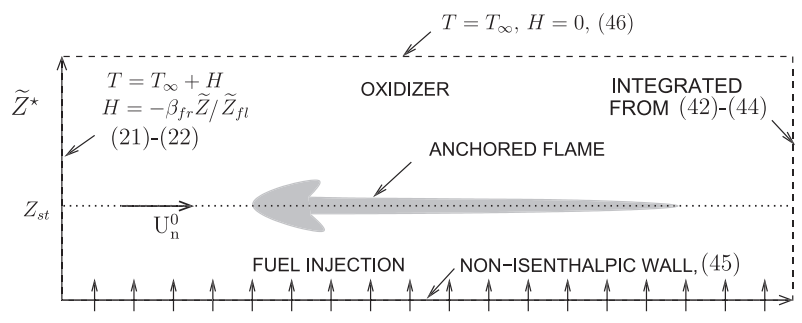

$n$

Fig. 6. The computational model problem used to study the influences of the cold porous wall on the propagation of straight edge flames. The boundary conditions indicated refer to equations in the main text. 


\subsection{Adiabatic triple-flame regimes}

For $\operatorname{Re}_{j} \geqslant O(1)$ the mass-transfer number is $\mathcal{B} \geqslant O(1)$ according to (9), the flame is far from the wall and the heat-loss term involving $\mathcal{B}$ in $(45)$ is negligible. The edge, trailing diffusion flame, and adiabatic flame temperatures are expected to be nearly equal, $T_{f} \sim T_{f l} \sim T_{f l}^{\infty}$. In these regimes, the Damköhler numbers of the edge and trailing diffusion flame are of the same order of magnitude, $\Delta_{f} \sim \Delta_{f l}$, and the flame is isenthalpic if the feed-stream temperatures are equal.

Fig. 7a-d shows the asymptotic propagation regimes found by using the scaling analyses outlined above for particular orderings of $\Gamma$, in the limit of large $\mathcal{B}$ and without streamwise hydrodynamic flow effects. The ordering of the reaction-zone shape (the shaded region) is different for the edge and for the trailing diffusion flame, and those orderings, following from the study of Daou and Liñán [24], depend on the ordering of $\Gamma$. The transverse extent of the edge reaction zone is of $O\left(\epsilon \delta_{M}\right)$ in all regimes, but the ordering of its longitudinal extent increases with increasing ordering of $\Gamma$, as does the ordering of the transverse extent of the reaction zone of the trailing diffusion flame. Advancing edges occur for small enough curvatures of the triple-flame, but there is a unique ordering of $\Gamma$, Fig. 7c, for which the edge propagation changes from advancing to retreating as the curvature increases (the value of $\Gamma$ in (38) increases). At larger ordering of $\Gamma$, Fig. 7d, just prior to the complete diffusion-flame extinction, all edges retreat.

Fig. 7e and $\mathrm{f}$ shows an example of a triple flame structure for the adiabatic case detailed above, and the results are quantitatively similar to those reported earlier [24] for the same value of the propagation-parameter $\Gamma$. The premixed front and the trailing diffusion flame burn more intensely as the stratification in the mixing layer is reduced. Extinction of the trailing diffusion flame is not observed in any of the advancing-edge regimes. The dynamics of triple flames in these regimes has been studied extensively in the past $[4,20-24,34]$.

\subsection{Non-adiabatic triple-flame regimes}

For $\operatorname{Re}_{j} \ll 1$ the mass-transfer number is $\mathcal{B} \ll 1$ according to (9), and the heat-loss term in (45) is non-negligible. In this regime, the flame is non-insenthalpic even in the case of equal feed-stream temperatures. The temperature of the edge $T_{f}$ and the trailing diffusion-flame temperature $T_{f l}$ given by (33) are not necessarily the same because of the heat losses to the disk surface. Similarly, $T_{f l}<T_{f l}^{\infty}$ in view of (25), (31), (33) and (34). Small differences between $T_{f}$ and $T_{f l}$ lead to the expression

$\frac{\Delta_{f}}{\Delta_{f l}} \sim \exp \left[-\frac{1}{\epsilon}\left(\frac{T_{f l}-T_{f}}{T_{f l} / T_{f}}\right)\right]=\exp \left[\frac{1}{\epsilon}\left(\frac{H_{f}+\beta_{f l}}{1-\left(H_{f}+\beta_{f l}\right) / T_{f}}\right)\right]$,

where $H_{f}=-\beta_{f l}+T_{f}-T_{f l}$ is a characteristic excess enthalpy in the edge region. In particular, if $T_{f}-T_{f l}=O\left(\epsilon^{n}\right)$ with $0<n<1$, then the excess enthalpy of the edge is larger than the excess enthalpy in the diffusion flame $H_{f}>-\beta_{f l}$ by amounts of order $\epsilon^{n}$, and $\Delta_{f} / \Delta_{f l}=O\left(\mathrm{e}^{\epsilon^{-|1-n|}}\right) \gg 1$. Similarly, if $T_{f}-T_{f l}=O\left(\epsilon^{n} \ln \epsilon\right)$ with $0 \leqslant n \leqslant 1$, then $H_{f}>-\beta_{f l}$ as well and $\Delta_{f} / \Delta_{f l} \sim \mathrm{e}^{\epsilon^{-|1-n|} /(1-n)} \gg 1$. These estimates show that, in the limit of large activation energy, small differences between the edge reaction-zone and trailing diffusionflame temperatures can lead to triple flames with large or orderunity front Damköhler numbers and small diffusion-flame Damköhler numbers, which are representative of propagating edges of diffusion flames that are close to extinction or have already undergone complete extinction. These edges propagate nearly adiabatically with an excess enthalpy distribution that, in the first approximation, is uniform in the n-direction and is given by (35); however phenomenologically different, such highly diffusive behavior of the excess enthalpy $H$ is somewhat reminiscent of the "Fife limit" encountered in earlier treatments of reactive-diffusive patterns, in which the controller variable is found to diffuse much more rapidly than the propagator variable [35].

Fig. $7 \mathrm{~g}-\mathrm{j}$ shows the asymptotic propagation regimes found by varying $\Gamma$ in (41) and (42) for negligible tangential curvature and without effects of the normal hydrodynamic flow to the front. The nearly adiabatic propagation of the edge is justified by the fact that, (i) for $\Gamma \ll 1 / \epsilon$, the transverse heat diffusion term $\Gamma \widetilde{\chi} \partial^{2} T / \partial \widetilde{Z}^{\star^{2}}$ in the preheat region, which is of $O\left(\Gamma \epsilon^{2}\right)$, is much smaller than the heat streamwisely convected to ignite the frozen mixture ahead, $\mathrm{U}_{\mathrm{n}}^{0} \partial T / \partial n=O(1 / \Gamma)$, which is balanced with the streamwise heat diffusion $\Gamma \partial^{2} T / \partial n^{2}=O(1 / \Gamma)$ there, and (ii) for $\Gamma \ll 1 / \epsilon$, the transverse heat diffusion in the edge reaction-layer region, $\Gamma \widetilde{\chi} \partial^{2} T / \partial \widetilde{Z} \star^{2}$, is of $O(\Gamma \epsilon)$ and much smaller than the streamwise diffusion $\Gamma \partial^{2} T / \partial n^{2}=O(1 / \Gamma \epsilon)$, which is balanced with the chemical reaction $O(1 / \Gamma \epsilon)$ there. Therefore the front propagates in a transverse adiabatic mode in the first approximation for a range of $\Gamma$, either advancing or very weakly retreating, $\Gamma \ll 1 / \epsilon$.

For propagation regimes in which $\Delta_{f} \mid \Delta_{f l} \gg 1$, extinction of the trailing diffusion flame exists when $\Delta_{f l} \lesssim \Delta_{E}$ and a balance between the transverse diffusion and strain rate has been established downstream of the edge. This qualitative difference from Fig. 7a-d is illustrated in Fig. $7 \mathrm{~g}-\mathrm{j}$. The diffusion-flame extinction occurs at dimensional distances from the edge of order $\mathrm{U}_{\mathrm{n}}^{0} \delta_{M}^{2} / D_{T}$ (with $\mathrm{U}_{\mathrm{n}}^{0}$ dimensional), which is obtained by a balance between transverse diffusion and streamwise convection, and in the present nondimensional notation corresponds to $n_{E} \sim \mathrm{U}_{\mathrm{n}}^{0} / \Gamma \epsilon^{2}$ in scales $\epsilon \delta_{M}$. Over distances of order $n_{E}$, the frozen profiles (21)-(24) are recovered. The dimensional time for extinction of the diffusion flame is therefore

$t_{E} \sim \delta_{M}^{2} / D_{T}$

which increases with decreasing $\Omega$.

These aspects of non-adiabatic triple-flame regimes can be observed in Fig. $7 \mathrm{k}$ and 1 , which shows the results of the numerical integration of (41)-(46) for small $\mathcal{B}$. The values employed for the computation in the model illustrated in Fig. 6 , namely $\Gamma=1.4$ and $\mathrm{Re}_{j}=0.08$, are beyond the static quenching point of the diffusion flame, as verified by a separate calculation of the extinction of the diffusion flame after dropping the streamwise transport terms in (41) and (42). The computation, which corresponds to a condition between that of Fig. $7 \mathrm{~h}$ and i, clearly exhibits a tripleflame type of structure for the propagating reaction layer, although some broadening in the preheat region is expected.

It is worth mentioning that diffusion-flame edges behind which diffusion flames cannot exist have been characterized previously within the context of non-equidiffusive non-premixed combustion [36], for which a non-zero excess enthalpy distribution is enhanced by non-unity Lewis-number effects. It is well known that, since the adiabatic temperature is a thermodynamic property that is independent of the diffusive properties of the mixture, the Lewis number affects the diffusion-flame temperature but does not influence the premixed-flame temperature [1]. Therefore, extinction of the trailing diffusion flame can be induced by increasing the average Lewis number of the mixture and thereby reducing the diffusion of reactants into the reaction zone, which decreases the diffusion-flame temperature while maintaining the same front temperature, in this way producing what have been termed "edges of flames that do not exist" [36]. The present situation is different in that the excess enthalpy is responsible for the extinction of the diffusion flame, this negative excess coming from the heat losses to the disk surface, which are larger in the presence of the diffusion flame than in the preheat and edge reaction-layer regions of the propagating edge flame. 
(a)

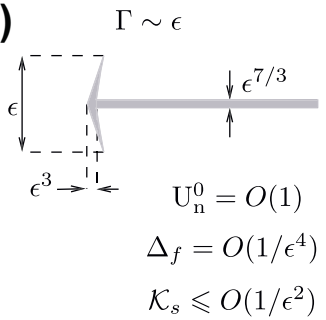

ADVANCING

(c)

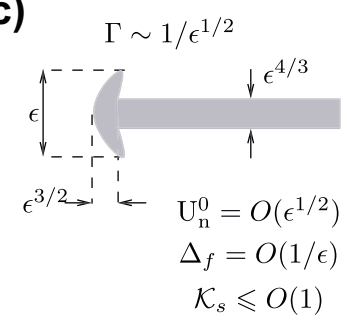

ADVANCING / RETREATING

(b)

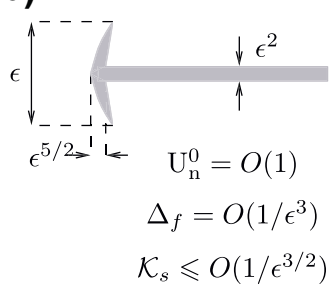

ADVANCING

(d)

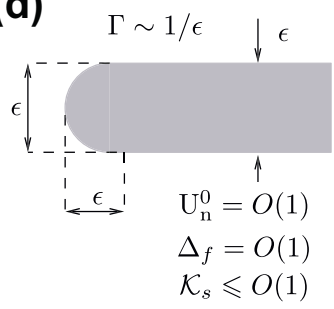

RETREATING (g)

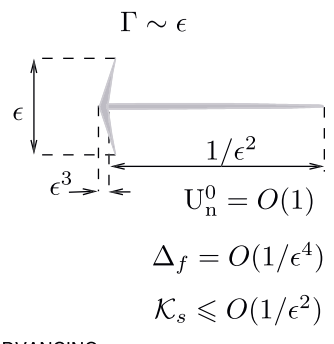

ADVANCING

(i)

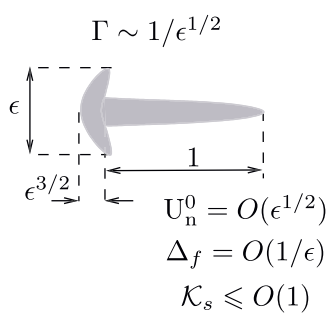

ADVANCING / RETREATING

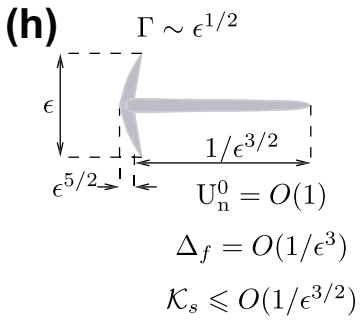

ADVANCING

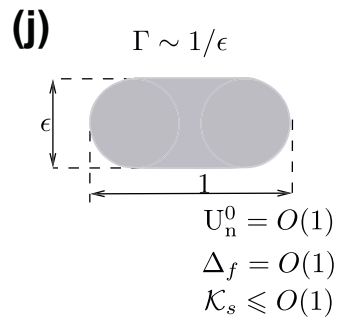

RETREATING
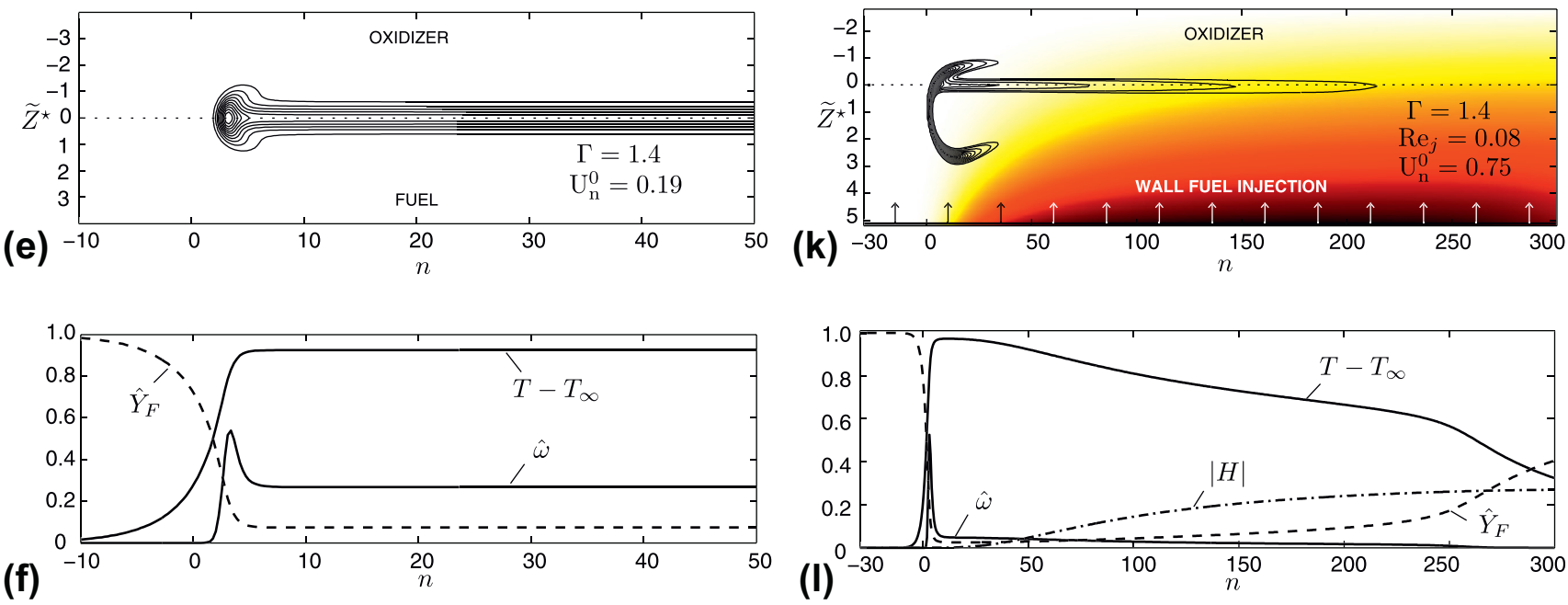

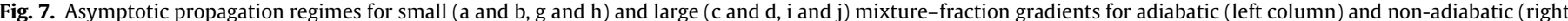

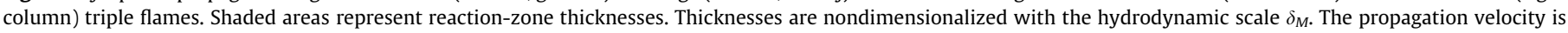

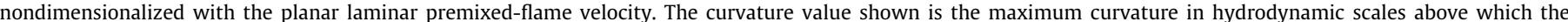

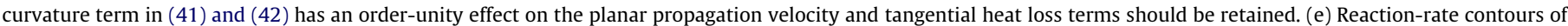

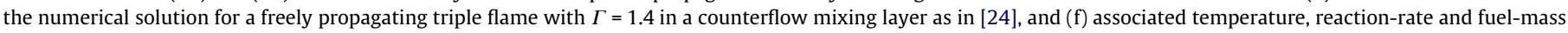

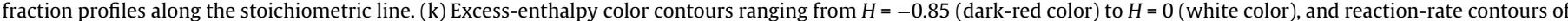

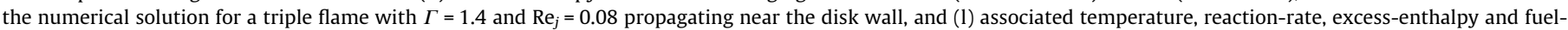
mass fraction profiles along the stoichiometric line.

\section{Edge dynamics}

When the kinematic relation (47) is substituted into the edge Eq. (37), the expression

$\frac{\partial G}{\partial t}+\left.\mathbf{v}_{\perp}\right|_{Z} \widetilde{Z}_{f l} \cdot \nabla G=\mathrm{U}_{\mathrm{n}}|\nabla G|$,

for the scalar $G$ is obtained, the three-dimensional version of which has been termed the "G-equation" in turbulent-combustion literature $[1,2]$. In this section, spatial coordinates and velocities are nondimensionalzed with $\delta_{M}$ and $\delta_{M} \Omega$, as in the original hydrodynamic formulation. A linear Markstein-type correction model for the effects of curvature on the propagation velocity $U_{n}$ [37] is used in this study to account for heat losses from the curved edge,

$\mathrm{U}_{\mathrm{n}}=\mathrm{U}_{\mathrm{n}}^{0}-\frac{\mathrm{Ma}}{\mathrm{Pr}} \mathcal{K}_{s}$
Here Ma, a Markstein number that in general would depend on the edge-flame structure, is set equal to unity to be consistent with the previous simplified formulation, which results in an equivalent Markstein length $\mathcal{L}=D_{T} / \mathrm{U}_{\mathrm{n}}^{0} \delta_{M} \Omega$, of the same order as the preheat thickness of the edge. The resulting approximation is expected to be reasonable for equidiffusional flames in analogy to wrinkled premixed flames [38], but to be limited to small differences $\left(U_{n}^{0}-U_{n}\right) / U_{n}^{0}$. Upon substituting (52) into (51), the G-equation reduces to

$\frac{\partial G}{\partial t}+\left.\mathbf{v}_{\perp}\right|_{\widetilde{Z}=\widetilde{Z}_{f l}} \cdot \nabla G+\frac{\mathcal{K}_{s}|\nabla G|}{\operatorname{Pr}}=\mathrm{U}_{\mathrm{n}}^{0}|\nabla G|$.

Expression (53) represents a Hamilton-Jacobi equation with a parabolic second-order differential operator in the curvature term. The first term on the left-hand side of (53) represents the local time variation of the edge shape, the second term accounts for the 
advection of the edge by the underlying swirling flow, the third term is a diffusive contribution that results from curvature effects on the propagation velocity, and the first term on the right-hand side is a source that accounts for the front self-propagating motion normal to itself because of the heat transfer from the planar edge to the frozen mixture upstream.

The scalar field $G$ is a assumed to be of the form $G(r, \theta, t)=F(r, t)-\theta$, with $\partial F / \partial \theta=0$, although it can be shown that the solution for $G$ is independent of this ansatz [2]. The normal vector to the front is then

$\mathbf{e}_{\mathbf{n}}=-\left[\left(r \frac{\partial F}{\partial r}\right) \mathbf{e}_{\mathbf{r}}-\mathbf{e}_{\theta}\right] / \sqrt{\left(r \frac{\partial F}{\partial r}\right)^{2}+1}$,

where use has been made of (36). Thus, the tangential curvature (48) can be expressed as

$\mathcal{K}_{s}=\left[r^{2}\left(\frac{\partial F}{\partial r}\right)^{3}+2 \frac{\partial F}{\partial r}+r \frac{\partial^{2} F}{\partial r^{2}}\right] /\left[r^{2}\left(\frac{\partial F}{\partial r}\right)^{2}+1\right]^{3 / 2}$.

In view of (2), by substituting (54) and (55) into (53), the equation

$$
\begin{gathered}
\frac{r \frac{\partial F}{\partial t}}{\sqrt{\left(r \frac{\partial F}{\partial r}\right)^{2}+1}}-\frac{\frac{\left|\phi_{f l}^{\prime}\right| r^{2}}{2} \frac{\partial F}{\partial r}-r V_{f l}}{\sqrt{\left(r \frac{\partial F}{\partial r}\right)^{2}+1}} \\
=-\frac{r^{2}\left(\frac{\partial F}{\partial r}\right)^{3}+2 \frac{\partial F}{\partial r}+r \frac{\partial^{2} F}{\partial r^{2}}}{\operatorname{Pr}\left[r^{2}\left(\frac{\partial F}{\partial r}\right)^{2}+1\right]^{3 / 2}}+\mathrm{U}_{\mathrm{n}}^{0}
\end{gathered}
$$

is obtained for the evolution of $F(r, t)$. In this formulation, $\left|\phi_{f l}^{\prime}\right| / 2$ and $V_{f f}$ represent the self-similar gradients in the radial direction of the radial and azimuth hydrodynamic velocity components, respectively, evaluated at the flame height $\widetilde{Z}=\widetilde{Z}_{f l}$. Similar equations have been encountered in earlier studies $[17,18,39]$ of spiral-wave propagation in pure reactive-diffusive systems; the results obtained in this section thereby represent a generalization by including swirling flow advection, in a form similar to that suggested earlier [40].

\subsection{Steadily rotating spirals}

For steadily rotating spirals, the front moves in a solid-rotation mode in the laboratory frame, describable as

$\mathbf{U}_{\mathbf{L F}}=-\omega r \mathbf{e}_{\theta}$,

and the functional form

$F(r, t)=f(r)+\omega t$,

can be substituted in (56), where $\omega$ is the angular velocity of the spiral (measured in the opposite direction from that of rotation of the disk) nondimensionalized with the disk angular velocity $\Omega$. An additional change of variables, $\varphi=r(d f / d r)=\tan \gamma$, where $\gamma$ is the slope angle between the edge and the radial unit vector, is performed here to facilitate calculations.

The experimental findings to be outlined in Section 5 suggest that the spirals rotate about a hole of finite radius, denoted dimensionally here by $r^{\star}$, which is observed to be much larger than a representative radius of curvature $\mathcal{L}$ in the vicinity of the tip. This limit is referred to as the "large core" limit in what follows in analogy to earlier works on spirals in reactive-diffusive systems [17]. As will be seen later in Fig. 15, the spiral flames spin in solid rotation motion for sufficiently small $\mathrm{Re}_{j}$ and $\mathrm{Da}$, and the radius $r^{\star}$ decreases with decreasing $\mathrm{Re}_{j}$ and increasing $\mathrm{Da}$. This is also true to some extent for slightly meandering spirals, for which the tip meanders about the disk center in an epicycloidal trajectory of an average radius that could be treated as $r^{\star}$ (see Section 5, Fig. 16). However, the quasi-steady decomposition (58) is not generally valid for meandering spirals, since the unsteady effects would be important in that case. The inner region $r-r^{\star}=O(\mathcal{L})$ represents the region where effects of tangential reactant and heat diffusion cause a non-negligible contribution to the front propagation velocity through the flame tangential curvature.

Spatial dimensions are renormalized in Eq. (56) for convenience as $\tilde{r}=r / \ell$, with the outer scale $\ell$ defined as

$\ell=\frac{2 \mathrm{U}_{\mathrm{n}}^{0}}{\Omega\left|\phi_{f l}^{\prime}\right|}$,

which corresponds to the radial length at which the radial velocity induced by the disk, $\left|\phi_{f l}^{\prime}\right| \Omega \ell / 2$, is equal to the dimensional planar front propagation velocity $\mathrm{U}_{\mathrm{n}}^{0}$. In this revised scale, the curvature (55) becomes

$\widetilde{\mathcal{K}}_{s}=\frac{1}{\tilde{r}} \frac{\varphi}{\left(1+\varphi^{2}\right)^{1 / 2}}+\frac{d \varphi / d \tilde{r}}{\left(1+\varphi^{2}\right)^{3 / 2}}$,

which is related to the propagation velocity by the Markstein formula (52), which becomes $U_{n}=U_{n}^{0}(1-\lambda \widetilde{\mathcal{K}})$ in these variables. Similarly, the transport Eq. (56) is transformed into

$\lambda\left[\tilde{r} \frac{d \varphi^{\prime}}{d \tilde{r}}+\left(1+\varphi^{2}\right) \varphi\right]=\left(1+\varphi^{2}\right)\left[\tilde{r}\left(1+\varphi^{2}\right)^{1 / 2}-\tilde{r}^{2}(\mathcal{S}-\varphi)\right]$,

where $\lambda$ is a dimensionless Markstein diffusivity that represents the ratio of the characteristic radius of curvature to the outer spatial scale,

$\lambda=\frac{\mathcal{L}}{\ell}=\frac{\left|\phi_{f l}^{\prime}\right|}{2 \operatorname{Pr}}\left(\frac{\delta_{M} \Omega}{\mathrm{U}_{\mathrm{n}}^{0}}\right)^{2} \ll 1$,

which is experimentally observed to be a small parameter, and where

$\mathcal{S}=2\left(V_{f l}+\omega\right) /\left|\phi_{f l}^{\prime}\right|$

is a swirl number that represents the ratio of the effective swirl in the Frenet frame to the radial velocity component at any radial distance.

Fig. 8 shows the variations of the swirl number $\mathcal{S}$ with the injection Reynolds number, calculated from (63) by using solutions of the von Kármán swirling flow with fuel injection, evaluated at the distance from the porous disk at which $Z=Z_{s t}=0.056$, the stoichiometric surface. The non-monotonicity of the dependence of $\mathcal{S}$ on $\operatorname{Re}_{j}$ for $\omega>0$, is a reflection of the non-monotonicity of the radial velocity illustrated in Fig. $4 a$. For small $\mathrm{Re}_{j}$, near $\mathrm{Re}_{j}^{\mathrm{min}}$, as the injection velocity is decreased the stoichiometric surface approaches the surface of the disk, and $\mathcal{S}$ becomes infinite because the radial velocity reaches zero when it is at the disk; on the other hand, for

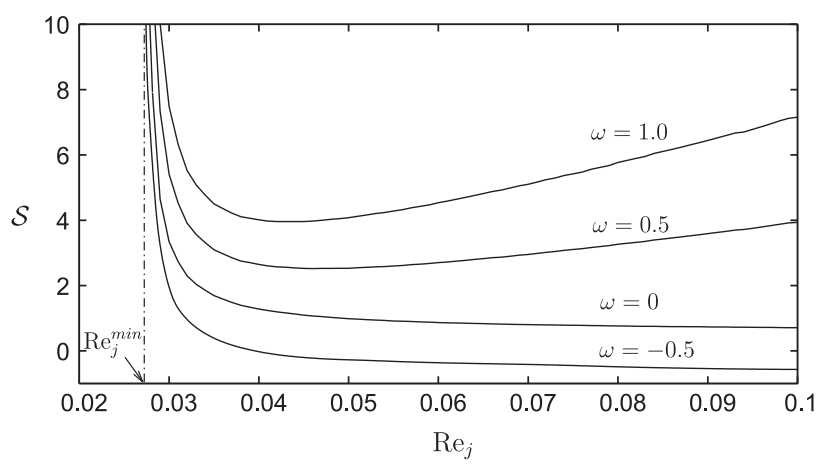

Fig. 8. The swirl number as a function of the injection Reynolds number for various values of the nondimensional angular velocity of the spiral. 
large $\mathrm{Re}_{j}$ the stoichiometric surface moves farther into the air, where the radial velocity is smaller, as the injection rate is increased.

An expression for the dimensionless core radius, which represents the lift-off radius at which the tip is anchored by the radial convection, in the approximation (52) of a linear dependency of the propagation velocity on curvature, can be obtained by rearranging (61), which gives

$\tilde{r}^{\star}=\left(1-\lambda \widetilde{\mathcal{K}}_{s}^{\star}\right) \frac{\sqrt{1+\varphi^{\star 2}}}{\mathcal{S}-\varphi^{\star}}$,

where the superindex ${ }^{\star}$ represents quantities evaluated at the tip. Eq. (64) shows that $\tilde{r}^{\star}=O(1)$ for $\lambda \ll 1, \mathcal{S}=O(1)$ and in the linear framework (52). The ratio $\ell / \delta_{M}=O\left(\Delta_{f}^{1 / 2}\right) \gg 1$ is a large number for advancing edges according to Fig. 7 and Eq. (40). This indicates that the typical core size is much larger than the mixing-layer thickness, as observed in the experiments. The Markstein length must be smaller than the mixing-layer thickness to have the preheat thicknesses smaller than $\delta_{M}$, so that $\mathcal{L} \ll \delta_{M} \ll r^{\star} \leqslant \ell$, which is achieved when $\Gamma \ll 1 / \epsilon$. In this limit, the spiral tip is anchored in a region of large radial and azimuth hydrodynamic velocities, which are of order $\ell \Omega$, whereas the normal fluid velocity to the disk is much smaller there, of order $\delta_{M} \Omega$.

\subsubsection{The outer region}

For $\tilde{r} \gg 1$ and $\lambda \ll 1$, the outer solution of $(61)$ is

$\varphi=\frac{\tilde{r}^{2} \mathcal{S}-\sqrt{\tilde{r}^{2}\left(1+\mathcal{S}^{2}\right)-1}}{\tilde{r}^{2}-1}$,

to leading order in $\lambda$, which represents the equation of a flame edge propagating with a velocity that is unaffected by curvature.

Substituting (65) into (64) for $\lambda=0$, the core radius

$\tilde{r}_{0}^{\star}=1 / \sqrt{1+\mathcal{S}^{2}}$

is obtained, which corresponds to the radius at which the magnitude of the incoming fluid velocity in the Frenet reference frame equals the planar propagation velocity, $\mathrm{U}_{\mathrm{n}}^{0}=\left\|\left.\mathbf{v}_{\perp}\right|_{\tilde{Z}_{Z}=\tilde{Z}_{f l}}-\mathbf{U}_{\mathbf{L F}}\right\|$, so that the edge arrives at the tip normal to the incoming relative flow. For $\tilde{r}<\tilde{r}_{0}^{\star}$, since $\left\|\left.\mathbf{v}_{\perp}\right|_{\tilde{Z}=\widetilde{Z}_{f}}-\mathbf{U}_{\mathbf{L F}}\right\|<\mathbf{U}_{n}^{0}$, the normal component of the relative swirling flow $\left(\left.\mathbf{v}_{\perp}\right|_{\tilde{Z}=\widetilde{Z}_{f l}}-\mathbf{U}_{\mathbf{L F}}\right) \cdot \mathbf{e}_{\mathbf{n}}$ is smaller than the propagation velocity $\mathrm{U}_{\mathrm{n}}^{0}$, so that the spiral must end at $\tilde{r}_{0}^{\star}$ in the first approximation $\lambda=0$.

For $\tilde{r} \rightarrow \infty$, the outer solution asymptotes to

$\varphi \sim \mathcal{S}-\frac{\sqrt{1+\mathcal{S}^{2}}}{\tilde{r}}+\frac{\mathcal{S}}{\tilde{r}^{2}}+O\left(\frac{1}{\tilde{r}^{3}}\right)$

It is worthwhile to mention that other reactive-diffusive patterns [10-13] mentioned in Section 1 display Archimedean spiral fronts far from the core, $r \sim \theta$, whereas in the present analysis Bernoulli spirals are obtained in the far field in the first approximation, $\tilde{r} \sim \mathrm{e}^{\theta / \mathcal{S}}$, which coincide with the streamlines of the two-dimensional flow field relative to the flame. The spiral growth in geometric progression found in the experiments suggests that this is in fact the case for the spiral flames, which explains the relatively few spiral turns observed and the rapid growth due to advection effects, departing from the linear Archimedean growth of the reactive-diffusive spirals found in earlier works [10-13].

For $\tilde{r} \sim \tilde{r}_{0}^{\star}$ the outer solution (65) asymptotes to

$\varphi \sim-\frac{1}{\mathcal{S}}+\frac{\sqrt{2}}{\mathcal{S}^{2}}\left(1+\mathcal{S}^{2}\right)^{5 / 4}\left(\tilde{r}-\tilde{r}_{0}^{\star}\right)^{1 / 2}+O\left(\tilde{r}-\tilde{r}_{0}^{\star}\right)$.

Fig. 9 shows the edges obtained by making use of(65) for different values of the swirl number. Large values of $\mathcal{S}$ are associated with highly curved spirals, whereas straight edges occur for small $\mathcal{S}$.

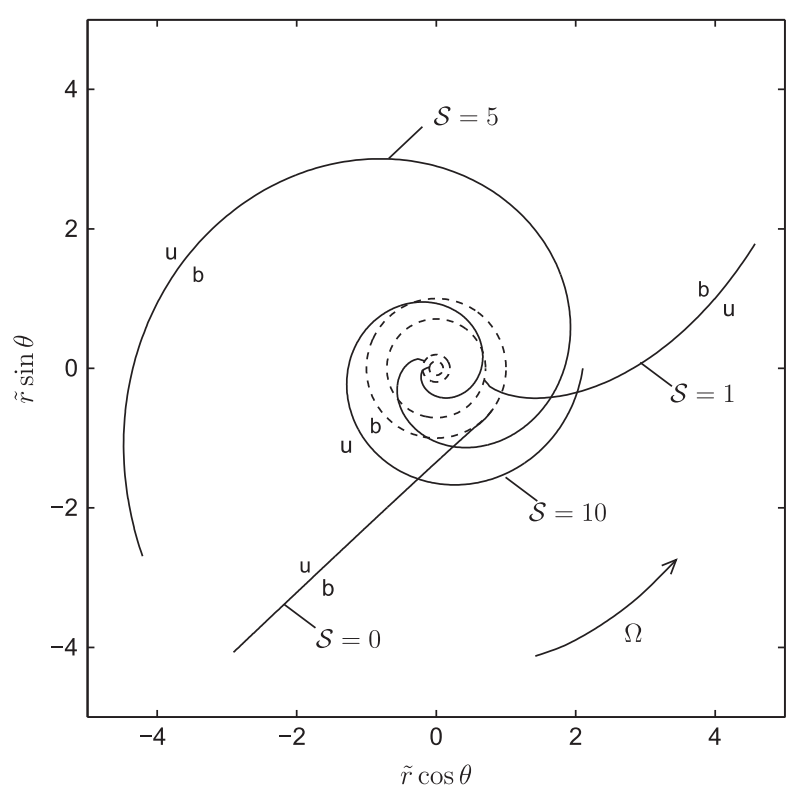

Fig. 9. Spiral edges obtained by using the outer solution (65). The letters $u$ and $b$ denote the unburnt and burnt sides of the edge respectively, and the arrow indicates the swirling direction of the hydrodynamic flow.

According to Fig. 8, by varying $\operatorname{Re}_{j}$ for the same value of $\mathcal{S}$, with $\mathcal{S}>0$, there is an infinite set of counter-rotating spirals, as well as an infinite set of co-rotating spirals at much lower values of $\operatorname{Re}_{j}$ (less than its value for $\omega=0$ ) that share the same edge geometry (since $\mathcal{S}$ is the same) and correspond to advancing fronts, $\mathrm{U}_{\mathrm{n}}^{0}>0$. In addition, at fixed $\mathrm{Re}_{j}$, achievable, for instance, by fixing $\Omega$ and the fuelinjection rate, there are correspondingly infinite sets of spirals with different shapes, each with a different $\mathrm{U}_{\mathrm{n}}^{0}>0$.

The second term in the expansion (68) is not differentiable at the spiral tip, and the curvature (60) becomes infinite there for $\mathcal{S}>0$, which renders the solution (65) non-uniform in the limit $\lambda=0$. For $\mathcal{S}=0$, a straight edge that is stationary in the incoming fluid reference frame $\left(\mathrm{U}_{\mathrm{n}}^{0}=0\right)$ is obtained, and it co-rotates with the local azimuth flow in the laboratory frame for all $\mathrm{Re}_{j}$ with an angular velocity $\Omega V_{f l}$ and a core radius $r^{\star}=\ell$ to every order in $\lambda$. However, single steady co-rotating edges have been observed experimentally only in multiple-spiral dynamics, a problem that is not addressed here. Additionally, the tip calculated by the outer solution (65) is found to be quasi-statically unstable to spatial perturbations, as detailed in Section 4.2.

\subsubsection{The inner region}

The non-uniformity of the outer solution at the tip leads to the existence of an inner region, $\tilde{r}-\tilde{r}^{\star}=O(\lambda)$, where the curvature term is non-negligible. According to Eq. (66), so long as $\mathcal{S}$ is not large, $\mathcal{S} \ll 1 / \lambda$, the core radius $r^{\star}$ is larger than the Markstein length $\mathcal{L}$, and $\lambda / \tilde{r}^{\star}$ can be regarded as a small parameter as well, which represents the large-core limit. In this limit, the inner-region equation

$\frac{d \varphi}{d \xi}=\left(1+\varphi^{2}\right)^{3 / 2}-\tilde{r}^{\star}\left(1+\varphi^{2}\right)(\mathcal{S}-\varphi)$,

is obtained to leading order in $\lambda / \tilde{r}^{\star}$, where

$\xi=\frac{\tilde{r}-\tilde{r}^{\star}}{\lambda}$,

is the inner variable. For $\xi \rightarrow+\infty$, the solution of (69) is asymptotic to $\varphi=-1 / \mathcal{S}+\Psi$, where $\Psi$ is a $O(\lambda)$ correction with respect to the outer leading-order value that satisfies the nonlinear equation $d \Psi / d \xi=\mathcal{S} \tilde{r}^{\star} \Psi^{2} / 2$ subject to $\Psi \rightarrow 0$ as $\xi \rightarrow+\infty$, which gives

$\varphi \sim-\frac{1}{\mathcal{S}}\left(1+\frac{2}{\tilde{r}^{\star} \xi}\right), \quad \xi \rightarrow+\infty$. 
For $\xi \rightarrow 0$, the inner Eq. (69) reduces to $d \varphi / d \xi \sim\left(\tilde{r}^{\star}-1\right) \varphi^{3}$ subject to $\varphi \rightarrow-\infty$ as $\xi \rightarrow 0$, which gives

$\varphi \sim-1 / \sqrt{2\left(1-\tilde{r}^{\star}\right) \xi}, \quad \xi \rightarrow 0$.

Upon substituting (72) into (60), the curvature value at the tip is found to be

$\widetilde{\mathcal{K}}_{s}^{\star} \sim \frac{1-\tilde{r}^{\star}}{\lambda}$

which represents the maximum curvature of the edge according to this small- $\lambda$ expansion. Under these conditions, the propagation velocity at the spiral tip obeys

$\frac{\mathrm{U}_{\mathrm{n}}^{\star}}{\mathrm{U}_{\mathrm{n}}^{0}} \sim \tilde{r}^{\star}$,

which represents a small fractional change $\left(U_{n}^{\star}-U_{n}^{0}\right) / U_{n}^{0}$.

Eq. (74) can be read in dimensional variables as $U_{\mathrm{n}}^{\star}=\Omega\left|\phi_{f l}^{\prime}\right| r^{\star} / 2$, which represents a balance between the edge propagation velocity (with curvature correction included) and the radial convection at $r^{\star}$. For $\lambda>0$, with $\lambda \ll 1$, the edge in the tip region is calculated to be normal to the radially advected mixture and advances towards it.

Fig. 10 shows examples of spiral tips obtained by the numerical integration of (61) subject to $\varphi \rightarrow \mathcal{S}$ at $\tilde{r} \rightarrow \infty$. All the spirals with $\lambda \neq 0$ arrive perpendicularly to a radial vector, so that the circular motion of the spiral is locally irrelevant at the core radius. As the dimensionless Markstein diffusivity $\lambda$ increases, the tip moves closer to the center of rotation, and it asymptotically passes through the center for a particular critical value $\lambda_{C}(\mathcal{S})$ that needs to be calculated numerically, and for which the edge goes from burning in an advancing mode, $U_{n}^{\star}>0$, to being locally stationary, $U_{n}^{\star}=0$. For $\lambda>\lambda_{c}$, there is an inner portion of the edge that propagates in a retreating mode, $U_{\mathrm{n}}^{\star}<0$, locally similar to a laboratory-stationary flame isola in a counterflow burner, while the outer portion still propagates in an advancing mode, as represented in Fig. 10 by the $\lambda=1.0$ spiral. These last behaviors, however, are unlikely to occur within the range of validity of the linear relationship (52), and they are not seen in any of the experiments.

The asymptotic expansions (68) and (71) do not match to second order. An intermediate region exists, of thickness $O\left(\lambda^{3 / 2}\right)$, that extends from the tip to the segment of the edge that is parallel to the radial unit vector, and the edge is purely azimuthally advected at that point. The analysis of this region provides corrections to the core radius, tip curvature and tip propagation velocity, as detailed

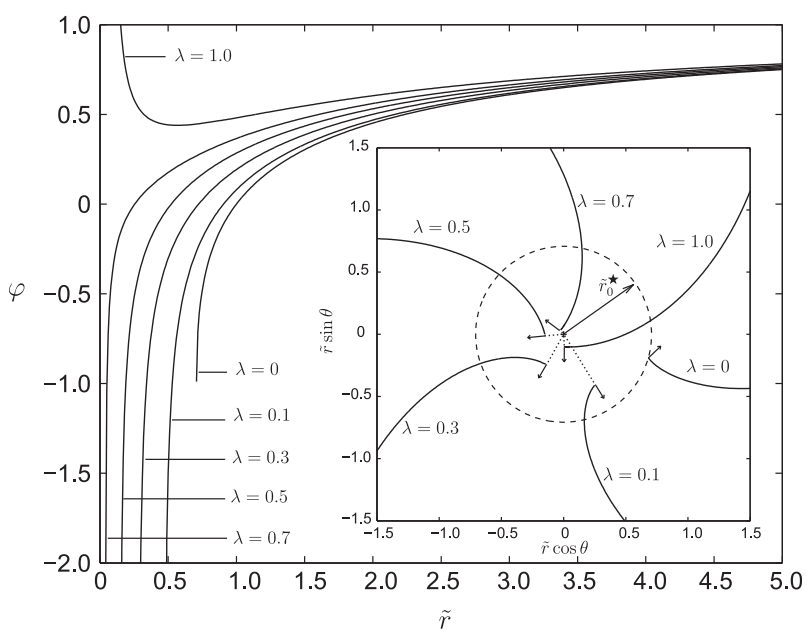

Fig. 10. Spiral edges obtained by the numerical integration of (61) for $\mathcal{S}=1$. in Appendix A. Eqs. (66) and (A.8) yield successive approximations for the spiral rotational velocity $\omega$ associated with a given core radius when the definitions (59), (62) and (63) are used.

\subsection{Spiral-flame anchoring and quasi-static stability}

The rate of variation $\Upsilon$ of the relative normal velocity component in the normal direction to the edge with respect to the propagation velocity variation may be defined as

$$
\begin{aligned}
\Upsilon & =\frac{1}{\mathrm{U}_{\mathrm{n}}^{0}}\left\{\left[\left(\mathbf{e}_{\mathbf{n}} \cdot \nabla\right)\left(\left.\mathbf{v}_{\perp}\right|_{Z=\tilde{Z}_{f l}}-\mathbf{U}_{\mathbf{L F}}\right)\right] \cdot \mathbf{e}_{\mathbf{n}}-\frac{\partial \mathbf{U}_{\mathbf{n}}}{\partial n}\right\} \\
& =-\frac{\varphi}{\sqrt{1+\varphi^{2}}}\left[\frac{(\mathcal{S}-\varphi)}{\sqrt{1+\varphi^{2}}}+\lambda \frac{d \widetilde{\mathcal{K}}_{s}}{d r}\right] .
\end{aligned}
$$

In the first equality, a general expression in the notation of the formulation (41)-(46), the normal derivative of $U_{n}$ is a curvature effect associated with the change in propagation velocity caused by the change in curvature of the edge that is produced by its infinitesimal displacement. The second equality translates the result into the notation of the present section.

The quantity $\Upsilon$ serves as a physical indicator of the stability of the front with respect to quasi-static infinitesimal perturbations in the normal direction. If $\Upsilon<0$, when the edge position is perturbed toward the propagation direction, the edge encounters a larger normal convective flow velocity component that returns it to its original position, as depicted in Fig. 11a and d. If $\Upsilon=0$ somewhere, that portion of the edge is in a neutrally stable state. If $\Upsilon>0$, when the edge position is perturbed toward the propagation direction, the edge encounters a smaller normal flow velocity component that cannot balance the propagation velocity; under these conditions the edge is unstable, as depicted in Fig. 11b and c, and it therefore is blown off from its original position, either towards or away from the disk center.

Edge-flame instabilities have been encountered in flame holes and flame disks in counterflow burners $[4,42]$, which correspond respectively to cases (b) and (c) in Fig. 11. Stationary flame holes and flame disks in the laboratory frame are found to heal or expand when perturbed in uniformly strained mixing layers close to the burner axis, and curvature variations of the edges with respect to their radial position produce propagation-velocity variations that contribute to further increase the value of $\Upsilon$ and enhance the instability process. Therefore, these structures can only be observed experimentally in stationary mode when they propagate to distances larger than the injector radius, where the radial velocity decays with the radial distance [43], as in Fig. 11a and d. However, in this analysis the curvature term $\partial \mathrm{U}_{\mathrm{n}} / \partial n=-\lambda \partial \widetilde{\mathcal{K}}_{s} / \partial n$ in (75) is found to benefit the stability of the spiral flames, since the curvature acts as a heat loss in the range of interest. In this range, the tip region behaves as a flame hole with the opposite curvature sign; the curvature perturbation of the displaced tip contributes to
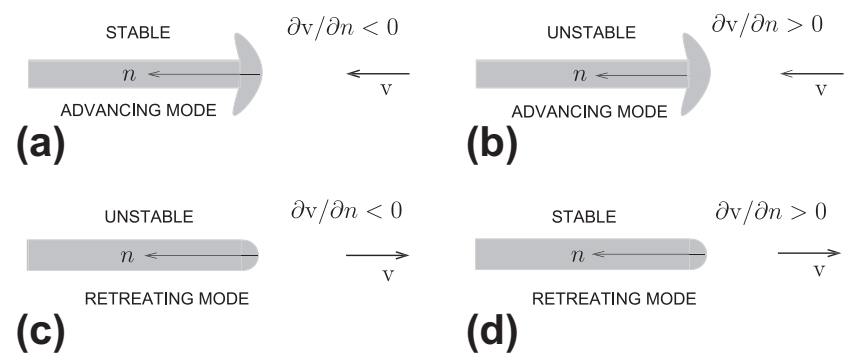

(b)

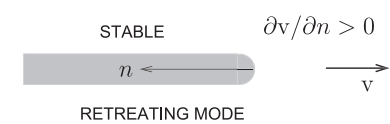

(d)

Fig. 11. Stability schematics of straight edge flames in the presence of normal convective velocity gradients $\partial \mathrm{v} / \partial n$. 
increase the propagation velocity in Fig. 11b for perturbations towards the burnt side, and to decrease the propagation velocity for perturbations towards the unburnt side.

Fig. 12a shows the calculated dependence of $\Upsilon$ on $\tilde{r}$ for $\lambda=0$. For $\tilde{r}>1 / \mathcal{S}$ with $\mathcal{S}>0$, the edge is stable as in Fig. 11a, and for $\tilde{r}^{\star}<\tilde{r}<1 / \mathcal{S}$, the inner portion of the edge and the spiral tip are unstable as in Fig. 11b. The point $\tilde{r}=1 / \mathcal{S}$ is neutrally stable since the normal variations are in the azimuth direction there, and the convective velocity modulus remains invariant in that direction. Edges with $\mathcal{S}=0$, which correspond to stationary fronts in the incoming fluid frame, are unstable for all $\tilde{r}$. Advancing edges propagating at a constant velocity thus are stable at large radii, become neutrally stable when the edge becomes tangent to the radial direction, and are unstable at smaller radii.

Fig. 12b shows the calculated variations of $\Upsilon$ with $\tilde{r}$ for $\mathcal{S}=1$ and $0 \leqslant \lambda \leqslant 1$. Consideration of fixed $\lambda$ and variable $\mathcal{S}$ produces similar dynamics. Nondimensional Markstein diffusivities in the range $0<\lambda<\lambda_{C}$ stabilize the tip region, compensating the decrease in the radial convection with decreasing radius by increasing the tip curvature, thus decreasing the tip propagation velocity. However, sufficiently large values of $\lambda$ or $\mathcal{S}$ produce a tip that propagates in a retreating mode, as shown in Fig. 10 for the case $\lambda=1.0$, which leads to an unstable tip as in Fig. 11c. As shown in the following section, spiral meandering is associated with large values of $\mathcal{S}$ that may enhance such loss of tip stabilization. In this linear quasi-static analysis, there is no combination of values of $\mathcal{S}$ and $\lambda$ for which $\Upsilon<0$ for all $\tilde{r}$, and there is always a small part of the edge that is unstable, which is close to the tip in hydrodynamic scales but far from it in scales of order $\mathcal{L}$.

Since the spirals observed in the experiments are stable, the above reasoning indicates that, according to this model, the curvature found in experiments must be large enough to produce departures from the linear Markstein formula (52) for the propagation velocity, such that the stabilizing term $\partial \mathrm{U}_{\mathrm{n}} / \partial n$ may actually be larger than $-\lambda \partial \widetilde{\mathcal{K}}_{s} / \partial n$. It seems likely that, in analogy with the effects of heat loss on laminar burning velocities [44,1], there is a critical
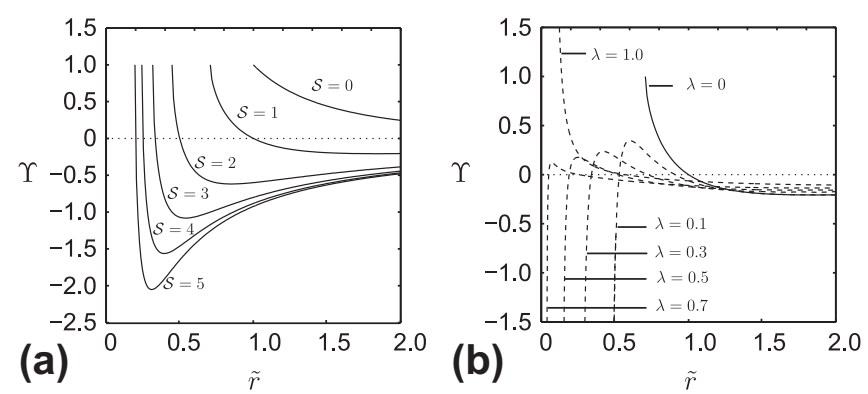

Fig. 12. (a) Quasi-static stability of spiral edges for $\lambda=0$. (b) Quasi-static stability of spiral edges for $\mathcal{S}=1$.

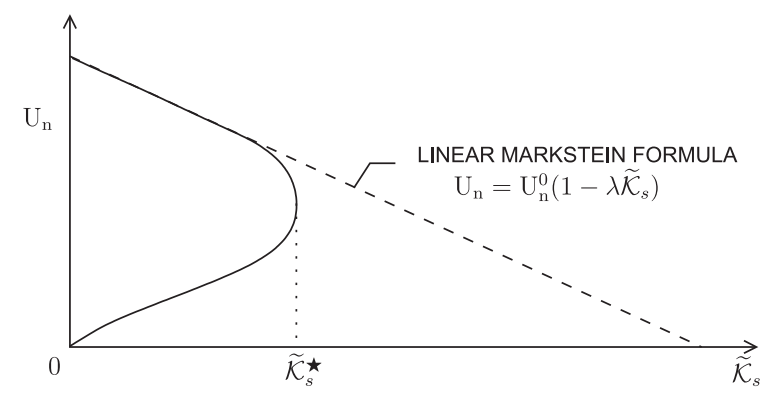

Fig. 13. Sketch of the edge propagation velocity as a function of the edge tangential curvature. finite value of the curvature at which the edge flame is extinguished, as illustrated schematically in Fig. 13. The non-linear effects beyond the range of the Markstein expansion may then stabilize the spiral up until the maximum curvature is reached, at which point the edge flame is extinguished. This condition would correspond to the tip of the spiral, where its curvature is maximum, and its location is at the minimum radius for spirals that rotate steadily.

\section{Experiments}

Experiments were conducted in the spinning, water-cooled, sintered, bronze porous-disk burner, depicted in Fig. 3 and described at the beginning of Section 2. The experimental arrangement is shown in Fig. 14. The burner assembly was mounted on a watercooled copper back-plate and a cup-shaped housing chamber which also serves as the plenum for the injected fuel gas. The fuel gas and the cooling water are supplied through concentric tubes located along the axis of this assembly. The concentric supply tubes are connected to external feed tubes through o-ring seals so that the entire burner assembly can be rotated around its axis with a stepper motor.

During an experiment, the burner is placed horizontally, with the exposed porous surface facing downwards to suppress buoyant instabilities, and it is then spun at a desired rotational speed with the cooling water supply turned on. Fuel gas is fed to the burner from a compressed-gas bottle through a programmable mass-flow controller at a specified flow rate and ignited by a propane torch. The entire experimental set up is enclosed in a large plexiglass box to prevent draft. All the flames observed in this study were blue and clearly visible to the naked eye. Flame images were captured using a $45^{\circ}$ mirror with a high-speed intensified-array video camera at 250 frames per second. Video images were digitalized by use of a frame grabber and then analyzed frame by frame using image-analysis routines. Although several dynamic flame patterns were observed in the experiments, as indicated in Fig. 15, results are presented only for the spiral flames. Table 1 summarizes the findings obtained from six different experimental data sets.

Fig. 15 shows the experimental map in the spiral-flame region $[5,7]$ obtained by varying the disk rotational speed and the fuel flow rate, dimensionally in part (a) and nondimensionalized in part (b), in terms of the Reynolds number of injection (3) and the reduced Damköhler number (40). The values for $\operatorname{Re}_{j}$ and $\Delta$ were calculated by using $S_{\mathrm{L}, \mathrm{st}}^{0}=40 \mathrm{~cm} / \mathrm{s}$ as a flame speed reference for $\mathrm{CH}_{4}$-air mixtures and the following physical properties that correspond to air at normal conditions: $\rho=1.19 \mathrm{~kg} / \mathrm{m}^{3}$ for the density, $v=1.57 \times 10^{-5} \mathrm{~m}^{2} / \mathrm{s}$ for the kinematic viscosity, $D_{T}=2.21 \times$ $10^{-5} \mathrm{~m}^{2} / \mathrm{s}$ for the thermal diffusivity, and $\mathrm{Pr}=0.71$ for the Prandtl number; the same physical properties are used throughout this section. A line of maximum rotational speed of the bulk of the spiral is seen in Fig. 15 to separate the meandering and solid-rotation regimes. The rotational speed decays on of both sides of that line.

In particular, in the meandering regime the tip undergoes an epicycloidal motion that is composed at least of two orbital motions and therefore two rotational frequencies. Such motion shares common characteristics with the meandering of reactive-diffusive spirals found in earlier works [17,18,39]; it is not well understood but believed to be influenced by system hysteresis. In the present experiments, tip meandering is associated with large swirl numbers, small radial advection and large spiral rotational velocities, as well as with increasing flame stand-off distances and decreasing strain rates and heat losses, which eventually produce transition from spiral flames to straight propagating edges and flame holes, as indicated in Fig. 15. The analysis of the meandering motion is beyond the scope of the present study. 


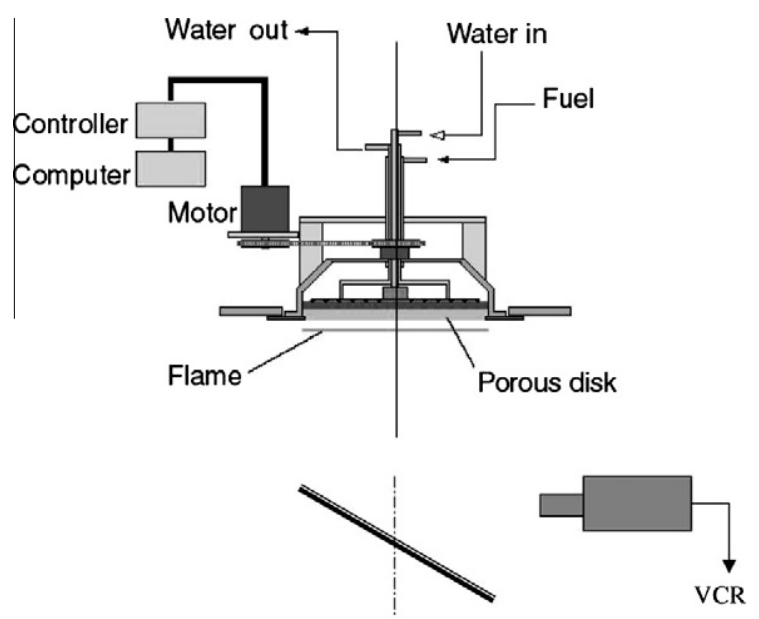

Fig. 14. Schematic illustration of the experimental apparatus.

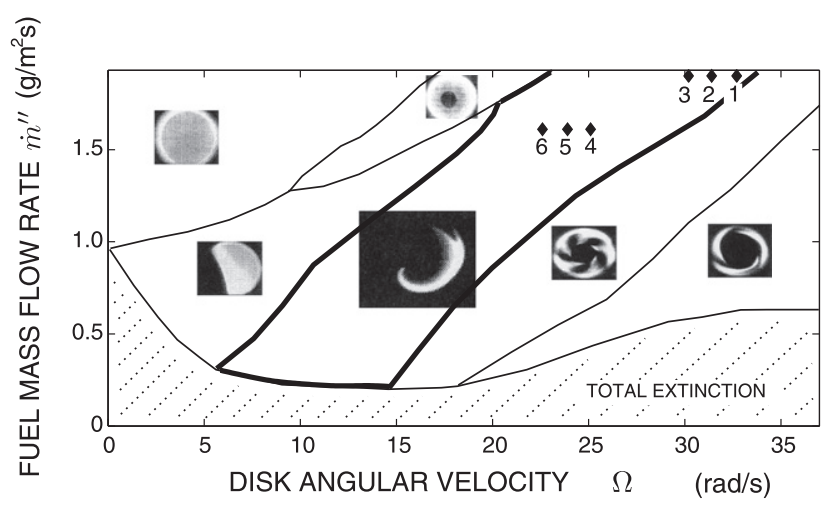

(a)

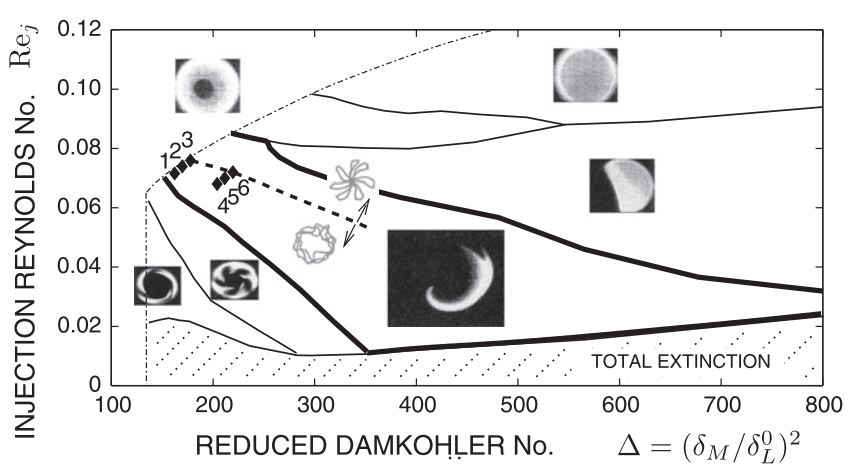

(b)

Fig. 15. (a) Dimensional and (b) nondimensional experimental pattern map focused on the spiral-flame region. The dashed line in (b) represents the line of maximum spiral rotational speed. The diamond-shaped points 1-6 correspond, respectively, to the experimental data sets $1-6$ in Table 1.
In the solid-rotation regime, the entire spiral structure undergoes a nearly circular motion of frequency $\omega$ and is anchored at a point of minimum radius $r^{\star}$ where the radial advection balances the normal edge propagation, as detailed in Section 4. In this regime, the rotational velocity decreases and the core radius increases approximately linearly with increasing disk velocities for a fixed fuel flow rate. As observed in Fig. 15, too large disk velocities or small fuel flow rates produce transition from this regime to the multiple-spiral regime, in which a number of spirals co-rotate with the flow. Six tests that best represent the steady-rotation regime were selected for further detailed analysis to compare with the present theory.

Fig. 16 shows the tip trajectories obtained from these six data sets, details of which are given in Table 1 . Slight meandering appears in cases 3 and 6 , where the disk velocity is minimum within the range, and the spiral velocity is near its maximum. While not exactly circular, most tip trajectories are close to that, and none exhibit clear petals for the cases selected.

Planar propagation velocities, calculated using expression (47) far from the disk center (at a distance about 3/4 of the disk radius, sufficiently large but away from disk-edge effects), are found to increase with increasing $\Gamma$, which is inversely proportional to the Damköhler number. These velocities are seen to be comparable with or somewhat less than the laminar burning velocity of a methane-air planar stoichiometric flame, $S_{L s t}^{0} \approx 40 \mathrm{~cm} / \mathrm{s}$, and as may be expected, they are smaller at smaller fuel injection rates. These values are used in (59) to calculate $\ell$. The propagation parameter $\Gamma$ is found to decrease with increasing spiral rotational velocities, since the mixing layer becomes thinner and the diffusion time through its thickness smaller. The values for $\Gamma$ obtained in Table 1 represent edge flames propagating somewhere between
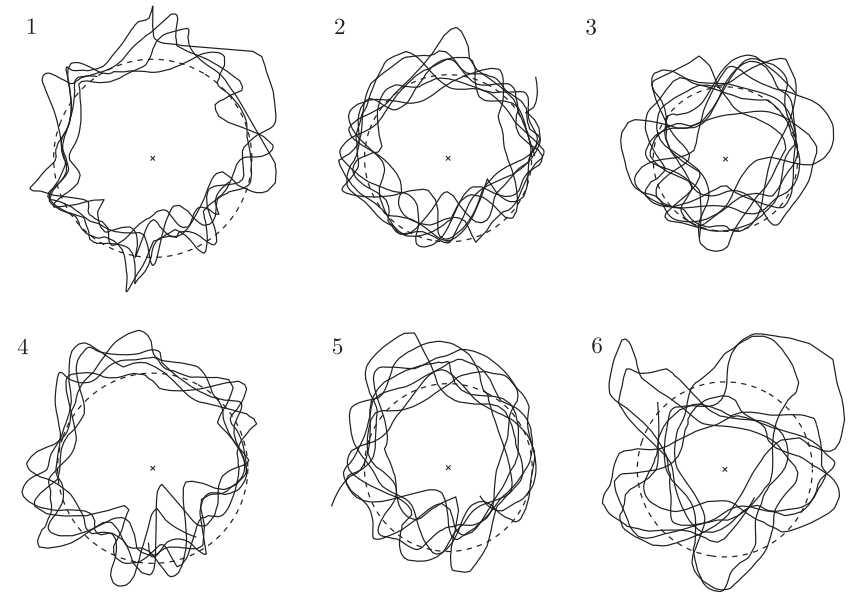

Fig. 16. Tip trajectories (solid line) and average core radius (dashed line) corresponding to the series of experiments (1-6) in Table 1.

Table 1

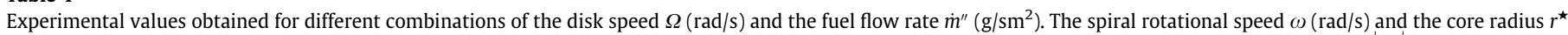

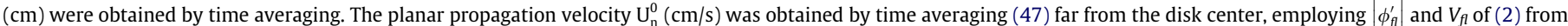

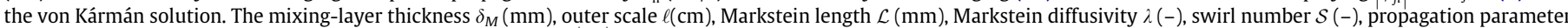

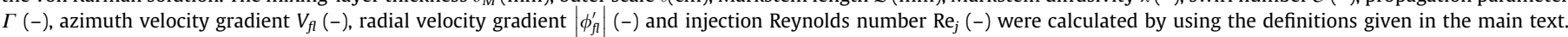

\begin{tabular}{|c|c|c|c|c|c|c|c|c|c|c|c|c|c|c|c|}
\hline Case & $\Omega$ & $\dot{m}^{\prime \prime}$ & $\omega$ & $\delta_{M}$ & $r^{\star}$ & $\mathrm{U}_{\mathrm{n}}^{0}$ & $\mathcal{L}$ & $\ell$ & $\operatorname{Re}_{j}$ & $\left|\phi_{f l}^{\prime}\right|$ & $V_{f l}$ & $\Gamma$ & $\mathcal{S}$ & $\lambda$ & $\lambda / \tilde{r}^{\star}$ \\
\hline 1 & 32.7 & 1.93 & 7.5 & 0.69 & 1.8 & 38.8 & 0.057 & 10.5 & 0.071 & 0.226 & 0.181 & 0.65 & 3.6 & 0.00054 & 0.0031 \\
\hline 2 & 31.4 & 1.93 & 11.3 & 0.71 & 1.5 & 39.1 & 0.056 & 11.2 & 0.073 & 0.222 & 0.176 & 0.63 & 4.8 & 0.00051 & 0.0038 \\
\hline 3 & 30.2 & 1.93 & 13.2 & 0.72 & 1.3 & 40.2 & 0.055 & 12.3 & 0.074 & 0.217 & 0.170 & 0.61 & 5.6 & 0.00045 & 0.0043 \\
\hline 4 & 25.1 & 1.61 & 8.2 & 0.79 & 1.6 & 29.4 & 0.075 & 9.8 & 0.068 & 0.239 & 0.194 & 0.56 & 4.3 & 0.00076 & 0.0046 \\
\hline 5 & 23.9 & 1.61 & 10.7 & 0.81 & 1.4 & 29.5 & 0.075 & 10.6 & 0.070 & 0.233 & 0.183 & 0.55 & 5.4 & 0.00071 & 0.0053 \\
\hline 6 & 22.6 & 1.61 & 11.9 & 0.83 & 1.5 & 29.9 & 0.074 & 11.7 & 0.072 & 0.226 & 0.182 & 0.53 & 6.3 & 0.00063 & 0.0048 \\
\hline
\end{tabular}




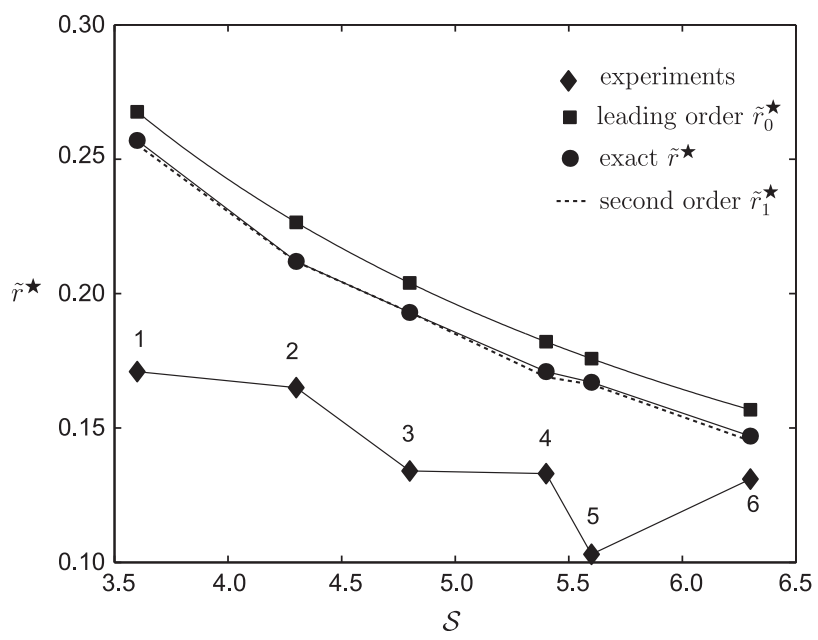

Fig. 17. Experimental and analytical values of the dimensionless core radius corresponding to the series of experiments (1-6) in Table 1.

the regimes (h) and (i) in Fig. 7, with a propagation velocity close to its adiabatic counterpart, supporting the results of Section 3.

Fig. 17 shows the resulting experimental nondimensional mean core radius $\tilde{r}^{\star}$ as a function of the swirl number. The theoretical results are also plotted in this figure for purposes of comparison. Farthest from the data is the leading-order result (66). The second-order result (A.8), which is almost identical to the exact value (64), is somewhat closer to the data. The theoretical and experimental dependencies on $\mathcal{S}$ are noticeably similar, and the fact that the theoretical values lie above the observation is consistent with the nonlinearity of the curve in Fig. 13, which becomes important as the extinction condition at the tip is approached, since the theoretical calculations do not include this effect.

Fig. 18 shows a comparison between the analytical front shapes, obtained by integrating (61) and the experimental results from Table 1. A good agreement is found for the overall trend, although the profiles seem to be somewhat inaccurate in the tip region, as expected. It is worth mentioning that, although the Markstein diffusivity and its ratio to the dimensionless core radius are found experimentally to be small, which support the theory proposed in Section 4, the typical propagation velocities in the neighborhood of the anchoring point are of order $r^{\star}\left|\phi_{f l}^{\prime}\right| \Omega / 2$, which, after numerical evaluation, represent an order-unity reduction with respect to the planar propagation velocity $\mathrm{U}_{\mathrm{n}}^{0}$. This further indicates that $\lambda \widetilde{\mathcal{K}}_{s}=O(1)$, and the Markstein linear correction (52) becomes innacurate for these large curvatures. The tip curvature $\widetilde{\mathcal{K}}_{s}^{\star}$, the tangential curvature for extinction of an edge flame, yet needs to be calculated numerically, although perhaps it could be approximated by the curvature for extinction of a positively curved two-dimensional premixed front [1].
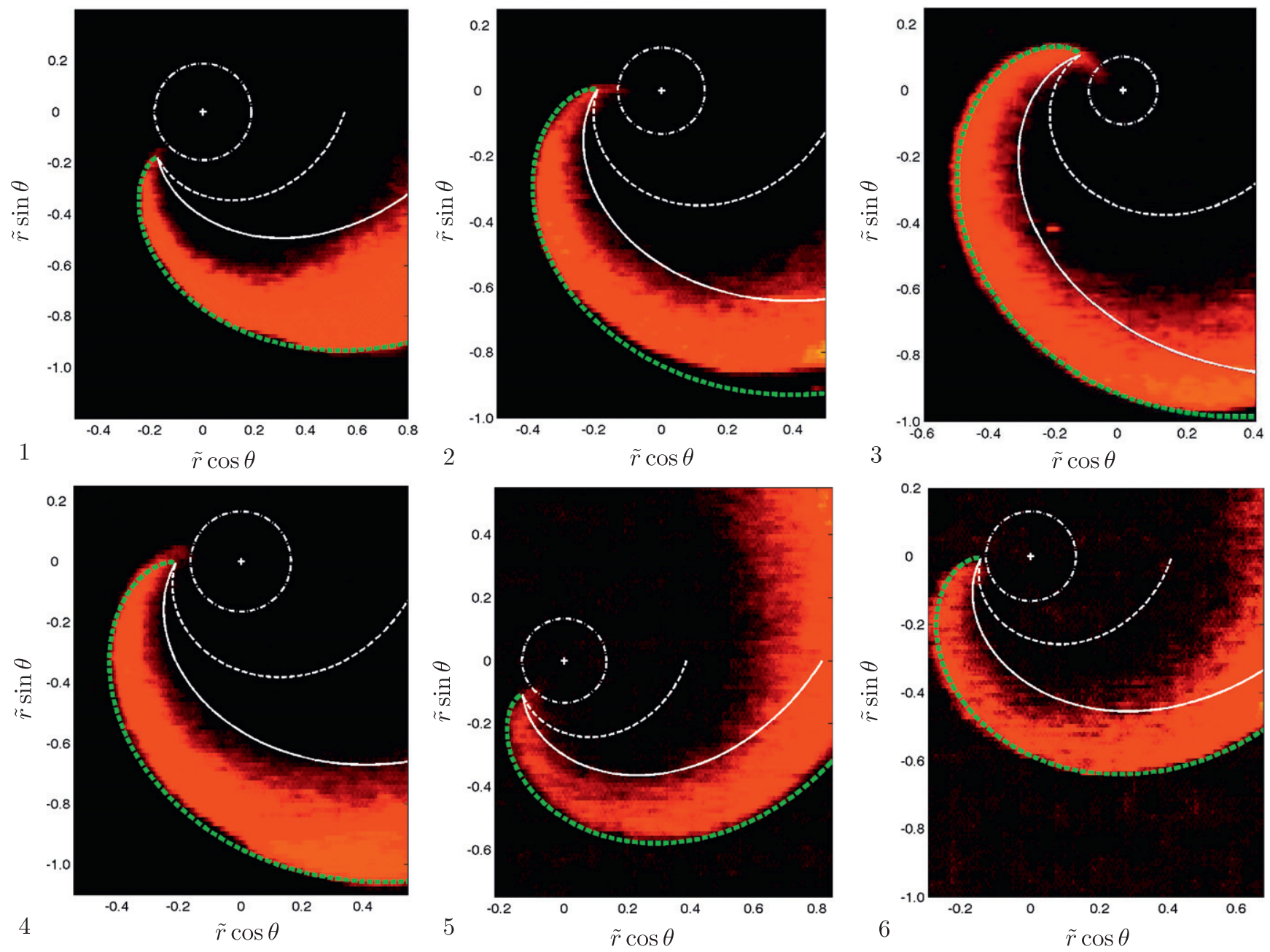

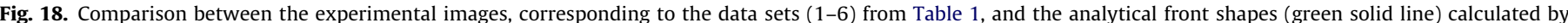

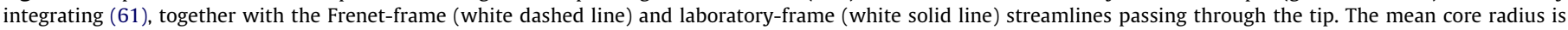
represented by the dot-dashed circle. 
The streamlines passing through the tip, both in the Frenet, $\tilde{r}=\tilde{r}^{\star} \exp \left[\left(\theta-\theta^{\star}\right) / \mathcal{S}\right]$, and in the laboratory reference frames, $\tilde{r}=\tilde{r}^{\star} \exp \left[\left(\theta-\theta^{\star}\right) /\left(\mathcal{S}-2 \omega / \Omega\left|\phi_{f l}^{\prime}\right|\right)\right]$, are also plotted in Fig. 18 . The Frenet-frame streamline passing through the tip clearly fails to describe the tail of the spiral, and since all the laboratory-frame streamlines have larger slopes than the former, the fluid particles in the stoichiometric coordinate must undergo extinction after being ignited. It may be considered that all fluid elements are required to have the same time for extinction, which is determined by heat losses to the disk as outlined in Section 3. With this idea, cases 1 and 3 of Table 1 were used to obtain the tail shape, as shown in Fig. 19. An array of fluid particles at the front at $t=0$
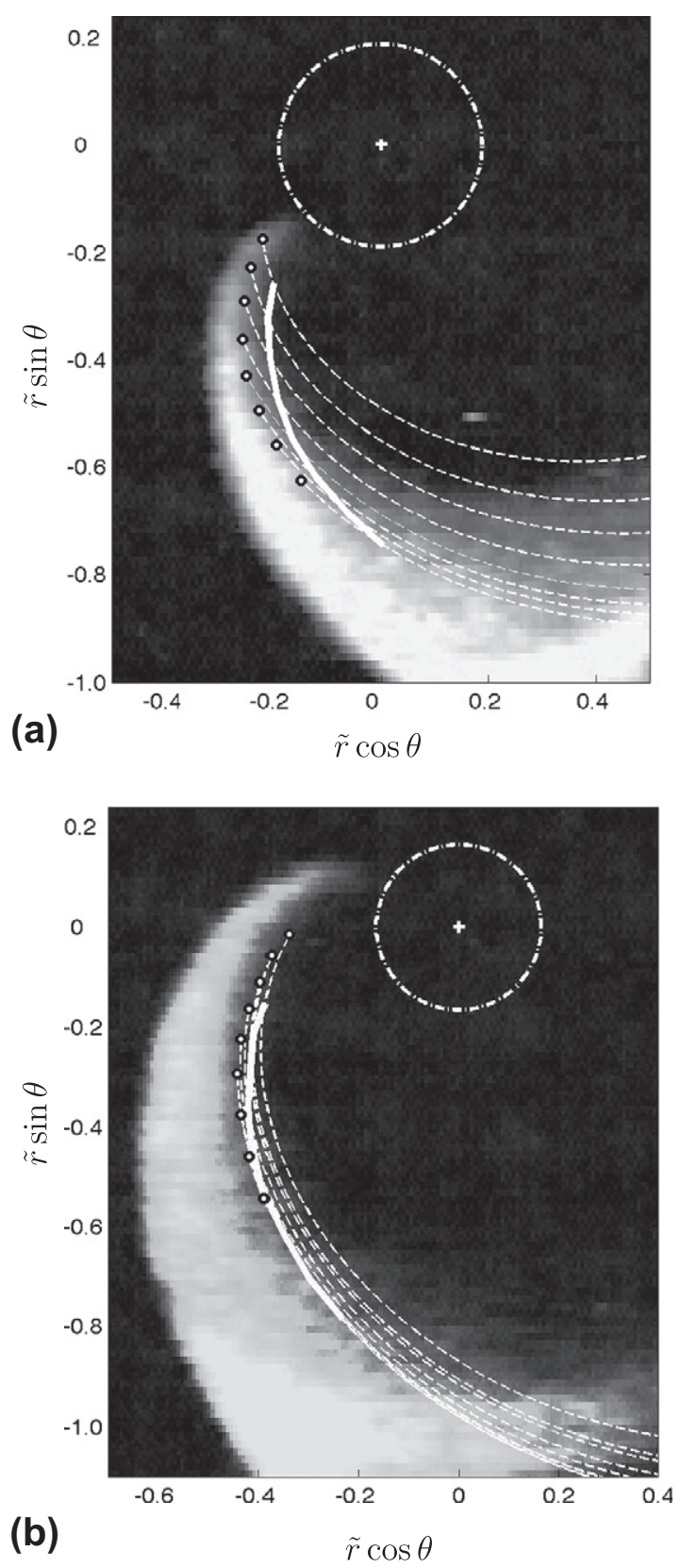

Fig. 19. Comparison between the experimental images at $t=t_{E}$ and the analytical tail shapes (white solid line) calculated by the theory of equal times of extinction, together with the laboratory-frame streamlines (white dashed line) emerging from the spiral front at $t=0$ along the stoichiometric plane. The points denote the fluid particles located on the front at $t=0$. Figure (a) corresponds to case 1 of Table 1 , which gives a $t_{E} \sim 40 \mathrm{~ms}$, and figure (b) corresponds to case 3 of Table 1 , which gives a $t_{E} \sim 68 \mathrm{~ms}$. was tracked along the laboratory-frame streamlines on the stoichiometric coordinate, and all particles extinguished after a time $t=t_{E}$, which increases with the Damköhler number, giving a good approximation of the tail shape; this calculation is justified as long as the entrainment remains small compared with the two planar velocity components, which appears to be a reasonable approximation along the flame radial positions encountered in this study. The extinction times are on the order of $50 \mathrm{~ms}$.

\section{Conclusions}

The propagation dynamics of spiral edge flames in von Kármán swirling flows was analyzed in this study. A formulation of the conservation equations was presented in the thermodiffusive and equidiffusive limits and within the framework of large overall activation energies. The formulation was written in terms of two main nondimensional parameters: the injection Reynolds number $\mathrm{Re}_{j}$ and the Damköhler number $\Delta$; these two numbers contained nondimensional combinations of the two experimentally controlled variables, the disk rotational velocity and the fuel flow rate, in addition to the chemical parameters. The resulting equations represented a non-adiabatic system with an induced nonzero excess enthalpy distribution, in which heat losses occurred because of the nearby cold disk surface. The Burke-Schumann and frozen regimes were summarized, and the diffusion-flame temperature was found to depend on the injection rate. The uniform diffusion flame is extinguished when $\Delta<\Delta_{E}$, with $\Delta_{E}$ a function of $\operatorname{Re}_{j}$.

A formulation in the Frenet frame, written in terms of the curvilinear intrinsic coordinates, was introduced to analyze propagating fronts that connect the burning and frozen regions. Asymptotic propagation regimes were obtained by scaling analyses of the conservation equations, and a two-dimensional numerical simulation of the conservation equations of a straight edge flame near a cold porous wall, embedded in a modelled von Kármán boundary layer, showed that there exist regimes, with Damköhler numbers below the static quenching Damköhler number $\Delta_{E}$ of the diffusion flame, for which a propagating front burns vigorously near the wall but the trailing diffusion flame is extinguished because of heat losses to the disk. That this behavior is possible is justified by noticing that the transverse heat diffusion in the edge reaction-layer and preheat regions is negligible compared to the streamwise balances of reaction-diffusion and convective-diffusion, respectively, in each region. As a result, the temperature along the stoichiometric line increases at the edge and gradually decays through the transition zone until the balance of strain and reaction is reached in the diffusionflame region, the point at which the reaction shuts off and the trailing flame is extinguished. The characteristic distance for extinction of the diffusion flame was found to be proportional to the propagation velocity and to increase with the Damköhler number of the edge. This defines a characteristic time $t_{E}$ for extinction of the diffusion flame, which increases with increasing flow times.

The steady dynamics of the spiral-edge propagation in the von Kármán hydrodynamic swirling field was analyzed by integrating the G-equation of the edge transport and using a Markstein-like expression for the tangential curvature correction of the propagation velocity. The dimensionless Markstein diffusivity $\lambda$, defined in (62) for this flow, becomes a singular perturbation parameter in the G-equation. The asymptotic structure of the edge was obtained by using asymptotic matching for $\lambda \ll 1$ in the large core limit, in which the radial distance of the tip from the center is small compared with the Markstein length, and solutions were found for spiral fronts that depended on this Markstein 
diffusivity and on the magnitude of the swirl number $\mathcal{S}$, which measures the ratio of the net swirl in the Frenet frame to the radial advection. Large values of $\mathcal{S}$ corresponded to highly curved spirals. Expressions were derived for the core radius as a function of $\mathcal{S}$ and $\lambda$, which in turn determines a relationship between the spiral rotational velocity and the core radius. In the absence of the decrease in propagation velocity produced by the Markstein diffusivity, the spirals were shown to become unstable at radii less than that at which the edge is tangent to the radial advection, but the increasing curvature stabilizes the edge propagation. This stabilization persists until the curvature reaches a critical maximum value at which edge-flame extinction occurs. This critical curvature for extinction determines the core radius and thereby the spiral rotational velocity. At the core radius, the edge is perpendicular to the radial direction, and its propagation velocity equals the radial gas velocity at the stoichiometric surface for steadily rotating spirals in solid rotation.

Experiments performed in the porous-disk burner were analyzed and theoretical predictions were tested. The spiral tip can undergo either a solid rotation motion for sufficiently large disk rotational velocities and small fuel flow rates, or epicycloidal motions for smaller disk rotational velocities and larger fuel flow rates. In the studied data range, the angular velocity of the spiral was found to decrease linearly with the disk rotational velocity for a given fuel flow rate. Meandering spirals are associated with rapidly rotating flames, large swirl numbers, moderately large injection Reynolds numbers, large Damköhler numbers, and decreasing heat losses and strain rates, which promote transition to the regimes of straight propagating edges and flame holes. Solid rotation is associated with the opposite behavior, which promotes transition to the multiple-spiral regime. Core radii were measured and agreed reasonably with the theoretical model, which predicts a reduction of the core radius with increasing values of $\mathcal{S}$. Spiral shapes obtained by the analytical model are in reasonable agreement with the experimental images.

These results show that non-unity Lewis numbers are not necessary for having spiral-shaped edges and cooling tails in these experiments, contrary to suggestions made in earlier works $[40,45,46]$.

The analyses performed in this study may be relevant for characterizing the dynamics of tangentially curved sheets of triple flames and their transport in fluid flows near non-adiabatic walls. Although much research has been performed on triple flame dynamics, there appear to be a large number of unknowns regarding the linear and non-linear effects of the tangential curvature and strain on the velocity of propagation of triple flames and their extinction. Finally, the meandering motion of the spiral flames reported in this study seems to emerge from an instability at the anchoring point of the tip, which may be worth investigating for having potential applications on the understanding of triple-flame anchoring processes in turbulent flows and on flame flickering. Meandering is the next theoretical problem to be addressed for spiral edges of diffusion flames in von Kármán swirling flows.

\section{Acknowledgments}

The authors are indebted to Professor A. Liñán for instructive conversations about this problem. The first author is also grateful to Professor P.A. Libby, Professor E. Fernández-Tarrazo, Dr. V. Kurdyumov, Professor J. Daou and Professor J.C. Prince for thoughtful suggestions on the numerical calculations, and to Professor J.C. Del Álamo's lab for the computational time. This work was partially supported by the NASA Microgravity Combustion Science Program.

\section{Appendix A. The intermediate region}

To bridge the region in which the expansions (68) and (71) do not match, the intermediate asymptotic expansion

$\varphi=-\frac{1}{\mathcal{S}}+\frac{\lambda^{1 / 3}\left(1+\mathcal{S}^{2}\right)^{2}}{\mathcal{S}^{3}} \psi+\cdots$,

may be defined, with the intermediate variable

$\sigma=\frac{\tilde{r}-\tilde{r}^{\star}}{\lambda^{2 / 3}}$

Substituting (A.1) and (A.2) into (61) and retaining terms of $O\left(\lambda^{2 / 3}\right)$, the equation

$\frac{d \psi}{d \sigma}-q \psi^{2}+\sigma+p=0$,

is obtained in the distinguished limit $\tilde{r}^{\star}-\tilde{r}_{0}^{\star}=O\left(\lambda^{2 / 3}\right)$. In this formulation, $p$ and $q$ are two order-unity constants given by

$p=\frac{\tilde{r}^{\star}-\tilde{r}_{0}^{\star}}{\lambda^{2 / 3}}$

and

$q=\frac{\left(1+\mathcal{S}^{2}\right)^{3 / 2}}{2 \mathcal{S}^{2}}$

Eq. (A.3) is a Ricatti equation, which can be cast into an Airy equation by changing the independent variable to $E=q^{1 / 3}(\sigma+p)$ and defining $M$ such that $\psi=-\left(1 / q^{2 / 3} M\right) d M / d E[41]$. In these variables, (A.3) becomes

$\frac{d^{2} M}{d E^{2}}=M E$,

the solution to which is a linear combination of Airy functions $\operatorname{Ai}(E)$ and $\operatorname{Bi}(E)$. Matching of (A.1) with (68) requires $M$ to be bounded for $E>0$, so that $M=C A \mathrm{i}(E)$, where $C$ is an integration constant. Therefore

$\psi=-\frac{1}{q} \frac{d \ln \operatorname{Ai}(\sigma)}{d \sigma}$

represents the intermediate solution.

For $E \rightarrow+\infty, M \sim \operatorname{Cexp}\left(-2 E^{3 / 2} / 3\right) /\left(2 E^{1 / 4} \pi^{1 / 2}\right)$ and $\psi \sim(\sigma / q)^{1 / 2}$; the substitution of this upper limit for $\psi$ into (A.1) exactly reproduces the second term of the expansion (68) of $\varphi$ in the upper overlapping region. For $\xi$ large, the intermediate expansion of (71) reads $\varphi \sim-1 / \mathcal{S}-\left(1+\mathcal{S}^{2}\right)^{2} / \mathcal{S}^{3} q \sigma$. Since $\operatorname{Ai}(E) \sim E-a_{1}$ near the first zero $a_{1}=-2.3381$ of Ai on the negative real axis, then $\psi \sim-q^{-2 / 3}\left[q^{1 / 3}(\sigma+p)-a_{1}\right]^{-1}$ for $E \sim a_{1}$, or equivalently, for $\sigma \rightarrow 0$. Therefore, asymptotic matching between the intermediate solution (A.1) and the inner solution (71) in the lower overlapping region requires that $p q^{1 / 3}=a_{1}$. This matching relation results in the second-order expansion

$\tilde{r}_{1}^{\star}=\tilde{r}_{0}^{\star}\left(1+2^{1 / 3} a_{1} \mathcal{S}^{2 / 3} \lambda^{2 / 3}\right)$

which shows that the tip radius undergoes reductions of order $\lambda^{2 / 3}$ with respect to its leading-order value when small curvature effects of $O(\lambda)$ on the edge propagation are considered.

Upon substituting the relation (A.8) into the Eqs. (73) and (74) of the inner region, the expansions

$\widetilde{\mathcal{K}}_{s}^{\star} \sim \frac{1-\tilde{r}_{0}^{\star}}{\lambda}-\frac{2^{1 / 3} c_{1} \mathcal{S}^{2 / 3} \tilde{r}_{0}^{\star}}{\lambda^{1 / 3}}$, 
and

$\frac{\mathrm{U}_{\mathrm{n}}^{\star}}{\mathrm{U}_{\mathrm{n}}^{0}} \sim \tilde{r}_{0}^{\star}\left(1+2^{1 / 3} c_{1} \mathcal{S}^{2 / 3} \lambda^{2 / 3}\right)$

are obtained.

\section{References}

[1] F.A. Williams, Combustion Theory, Addison-Wesley, 1965.

[2] N. Peters, Turbulent Combustion, Cambridge University Press, 2000.

[3] R. Yetter, I. Glassman, H.C. Gabler, Proceedings of the Combustion Institute 28 (2000) 1265-1272.

[4] J. Buckmaster, Progress in Energy and Combustion Science 28 (2002) 435-475.

[5] V. Nayagam, Pattern Formation in Diffusion Flames Embedded in Von Kármán Swirling Flows, Tech. Rep. NASA/CR-2006-214057, National Aeronautics and Space Administration, 2006.

[6] V. Nayagam, F.A. Williams, Dynamics of spiral flames in von Kármán swirling flows, in: 31st International Symposium in Combustion, 2006 (poster).

[7] J. Urzay, V. Nayagam, F.A. Williams, Proceedings of the Combustion Institute 32 (2009) 1219-1226.

[8] K.N. Hossain, T.L. Jackson, J. Buckmaster, Proceedings of the Combustion Institute 32 (2009) 1209-1217.

[9] V. Nayagam, F.A. Williams, Physical Review Letters 84 (3) (2000) 479-482.

[10] T. Plesser, S. Muller, B. Hess, Journal of Physical Chemistry 94 (1990) 75017507.

[11] M. Bär, S. Nettesheim, H. Rotermund, M. Eiswirth, G. Ertl, Physical Review Letters 74 (1995) 1246-1254.

[12] F. Siegert, C. Weijer, Current Biology 5 (1995) 937-943.

[13] D.E. Clapham, Cell 80 (1995) 258-259.

[14] Y. Zel'dovich, D.A. Frank-Kamenetskii, Acta Physicochimica URSS 9 (2) (1938) 341-350.

[15] A. Kolmogorov, I. Petrovskii, N. Piskounov, Bulletin of the State University of Moscow 1 (1937) 1-25.

[16] P. Grindrod, The Theory and Applications of Reaction-Diffusion Equations: Patterns and Waves, Clarendon Press, Oxford, 1996.

[17] J.J. Tyson, J.P. Keener, Physica D 32 (1988) 327-361.

[18] J. Keener, J. Sneyd, Mathematical Physiology, Springer, 2004.

[19] A. Liñán, Acta Astronautica 1 (7-8) (1974) 1007-1039.

[20] H. Phillips, Proceedings of the Combustion Institute 10 (1965) 1277-1283.

[21] J.W. Dold, Combustion and Flame 76 (1989) 71-88.
[22] P.N. Kioni, B. Rogg, K.N.C. Bray, A. Liñán, Combustion and Flame 95 (1993) 276-290.

[23] G.R. Ruetsch, L. Vervisch, A. Liñán, Physics of Fluids 7 (1995) 1147-1454.

[24] J. Daou, A. Liñán, Combustion Theory and Modelling 2 (1998) 449-477.

[25] A. Fan, S. Minaev, E. Sereshchenko, R. Fursenko, S. Kumar, W. Liu, K. Maruta, Proceedings of the Combustion Institute 32 (2009) 3059-3066.

[26] G. Jomaas, J.K. Bechtold, C.K. Law, Proceedings of the Combustion Institute 31 (2007) 1039-1046.

[27] H.G. Pearlman, P.D. Ronney, Journal of Chemical Physics 101 (1994) 26322633.

[28] T. von Kármán, Zeitschrift für Angewandte Mathematik und Mechanik 1 (1921) 233-252

[29] J. Urzay, Theoretical Studies in Spiral Edge-flame Propagation and Particle Hydrodynamics, Ph.D. Thesis, University of California San Diego, 2010.

[30] C.E. Weatherburn, Differential Geometry of Three Dimensions, Cambridge University Press, 1961.

[31] P. Pelce, J. Sung, Physical Review A 44 (1999) 7906-7909.

[32] E. Fernández-Tarrazo, A.L. Sánchez, A. Liñán, F.A. Williams, Combustion and Flame 147 (2006) 32-38.

[33] V. Kurdyumov, E. Fernández-Tarrazo, Combustion and Flame 128 (2002) 382394.

[34] E. Fernández-Tarrazo, M. Vera, A. Liñán, Combustion and Flame 144 (2006) 261-276.

[35] A.J. Bernoff, Physica D 53 (1991) 125-150.

[36] R.W. Thatcher, J.W. Dold, Combustion Theory and Modelling 4 (2000) 435457.

[37] G.H. Markstein, Journal of Aeronautical Sciences 18 (1951) 199-209.

[38] P. Clavin, F.A. Williams, Journal of Fluid Mechanics 116 (1982) 251-282.

[39] V. Hakim, A. Karma, Physical Review E 60 (1999) 5073-5105.

[40] F.A. Williams, Spiral diffusion flames, in: F.J. Higuera, J. Jiménez, J.M. Vega (Eds.), Simplicity, Rigor and Relevance in Fluid Mechanics: A volume in honor of Amable Liñán, CIMNE, Barcelona, 2004, pp. 61-74.

[41] C.M. Bender, S.A. Orszag, Advanced Mathematical Methods for Scientists and Engineers, Springer, 1999.

[42] V. Nayagam, R. Balasubramaniam, P.D. Ronney, Combustion Theory and Modelling 3 (1999) 727-742.

[43] V.S. Santoro, A. Liñán, A. Gomez, Proceedings of the Combustion Institute 28 (2000) 2039-2046.

[44] G. Joulin, P. Clavin, Acta Astronautica 3 (1976) 223-240.

[45] V. Nayagam, F.A. Williams, Journal of Fluid Mechanics 458 (2002) 219-228.

[46] V. Nayagam, F.A. Williams, Combustion Science and Technology 176 (2004) 2125-2142. 